\title{
Small rings in the bigger picture: ring expansion of three- and four-membered rings to access larger all-carbon cyclic systems
}

\author{
Bohdan Biletskyi, Pierre Colonna, Kévin Masson, Jean-Luc Parrain, \\ Laurent Commeiras (iD * and Gaëlle Chouraqui (D) *
}

\begin{abstract}
The release of the inherent ring strain of cyclobutane and cyclopropane derivatives allows a rapid buildup of molecular complexity. This review highlights the state-of-the-art of the ring expansions of threeand four-membered cycles and is organised by types of reactions with emphasis on the reaction mechanisms. Selected examples are discussed to illustrate the synthetic potential of this elegant synthetic tool.
\end{abstract}

\section{Introduction}

The importance of step economy towards the construction of molecular diversity is still a challenge that the synthetic organic chemists' community is eager to take up. ${ }^{1}$ On the other hand, a major advantage of small rings is their inherent ring strain ${ }^{2}$ and, consequently, their high reactivity. The reported values of the strain energy, viz. $27.5 \mathrm{kcal} \mathrm{mol}^{-1}$ for cyclopropane and $26.5 \mathrm{kcal} \mathrm{mol}^{-1}$ for cyclobutane $\left(0 \mathrm{kcal} \mathrm{mol}{ }^{-1}\right.$ for the benchmark cyclohexane), clearly set the stage for ring-opening through strain relief. $^{3}$ This release of ring tension allows for a scalable, rapid and

Aix Marseille Univ, CNRS, Centrale Marseille, iSm2, Marseille, France. E-mail: gaelle.chouraqui@univ-amu.fr controlled synthetic access to the expected target molecules. ${ }^{4}$ In particular, these methods are applicable to propose new fragments in "Diversity Oriented Synthesis (DOS)" of fine chemicals and materials. ${ }^{5}$

This review aims to provide a recent account on the ring expansion of cyclobutyl and cyclopropyl derivatives to access larger membered all-carbon rings. Thanks to their inherent ring strain, small rings represent a valuable three- or fouratoms building element in the search to reach molecular complexity in an atom economical manner. Rearrangements, ${ }^{6}$ in that perspective, are an opportunity for small size rings to participate in fascinating chemical transformations. In addition, rearrangements of small rings are not only ruled by

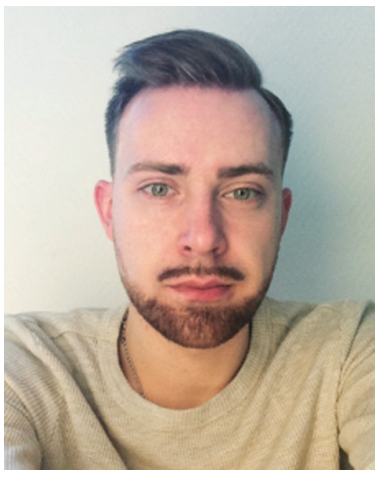

Bohdan Biletskyi
Bohdan Biletskyi began his education as an organic chemist in 2009 at the National Technic University of Ukraine (Kyiv). He received his $P h D$ degree in organic chemistry in 2018 from AixMarseille University under the guidance of Dr Hervé Clavier and Dr Alphonse Tenaglia. Then, he pursued his research as postdoctoral fellow. He first worked with Dr Gaëlle Chouraqui and Prof. Laurent Commeiras on the reactivity of a new class of donoracceptor cyclopropanes. He recently joined Dr Xavier Bugaut's team, in 2020. His current research interests center on the study of new type of organocatalysts with Halogen-Hydrogen bond activation.

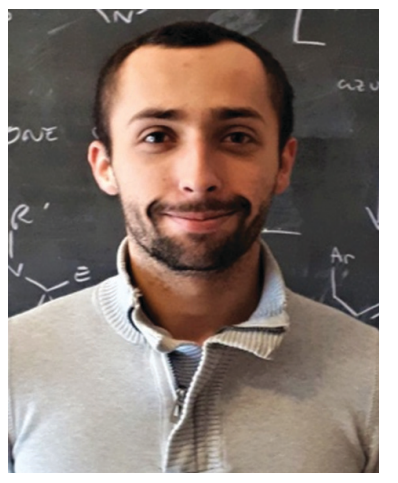

Pierre Colonna
Pierre Colonna studied chemistry at Université Paris-Saclay and received a joint-honours MSc degree in 2018 from Université Paris-Sud and Ecole Polytechnique. $\mathrm{He}$ is currently pursuing his $P h D$ under the supervision of Dr Gaëlle Chouraqui and Prof. Laurent Commeiras at AixMarseille University (France). His research focus on the synthesis of benzocyclobutene derivatives from a new class of donor-acceptor cyclopropanes. 
stereoelectronic considerations but also, by steric effects. For those reasons, high stereoselectivity often arise from such approaches. Although the ring enlargement of cyclopropyl and cyclobutyl derivatives was covered in different reviews, none was available on the recent progress made on this specific topic. For instance, Njardarson disclosed in 2013, the metalcatalysed ring enlargement of three- and four-membered rings. ${ }^{7}$ In 2011, Cramer gave an account on cyclobutanes in catalysis. ${ }^{8}$ The ring expansion strategy as a key step in the total synthesis of natural products has also been covered several times but it is either old ${ }^{4}$ or quite specific to a particular molecular species (i.e. cyclopropanols). ${ }^{9}$ Cyclobutanols and cyclopropanols, as a matter of fact, have been the focus of very recent attention. ${ }^{9,10}$ The purpose of this review is to give an overview of the progress made since 2012 and collected up until December 2020.

Ring expansion of small carbocyclic systems can be classified into three main groups of rearrangements (Scheme 1), and the following discussion was structured according to these three types of reactions: ${ }^{11}$

- Group 1: carbon(s) insertion through opening of a bicyclic system, instigated by functional groups decorating the ring,

- Group 2: carbon(s) insertion through migration to an exocyclic group,

- Group 3: electrocyclic and sigmatropic reactions.

We wish to critically discuss the scope, applications, mechanisms and the potential for future development of the title species. It is not intended to give here, a comprehensive

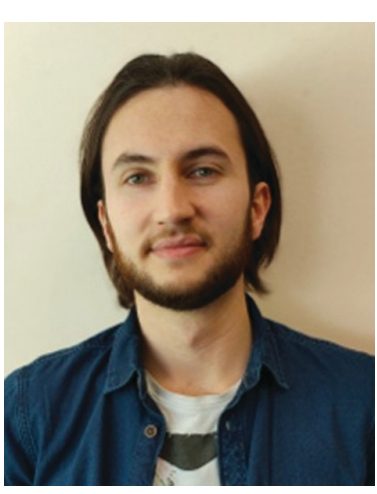

Kévin Masson
Kevin Masson studied chemistry at the Ecole Nationale Superieure de Chimie de Mulhouse where he obtained his bachelor's degree in 2016. After a one-year industrial experience within the Servier Research Institute of Medicinal Chemistry in Budapest, he completed both a chemical engineering and a MSc degree in organic chemistry. After his graduation, he moved to AixMarseille University where he is currently working towards his PhD under the supervision of Dr Gaëlle Chouraqui, Dr Laurent Commeiras and Dr Paola Nava. His research interests focus on the development of new methods to access highly substituted all-carbon medium sized rings.

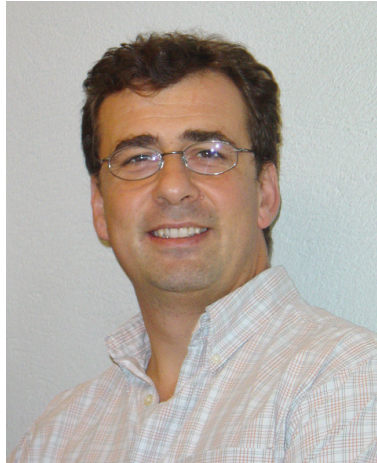

Jean-Luc Parrain

Jean-Luc Parrain obtained his $\mathrm{PhD}$ in Chemistry at the University of Nantes (France) under the supervision of Prof. Jean-Paul Quintard. After postdoctoral studies in the laboratory of Prof. Steve Davies at the University of Oxford (UK), he joined the CNRS in 1990 and he moved to Aix Marseille University in 1995. His research interests include new catalytic reactions toward new synthetic methods, development of organo-element (Sn, Si and B) reagents, total synthesis of natural compounds and, more recently, spatially resolved functionalization of surfaces.

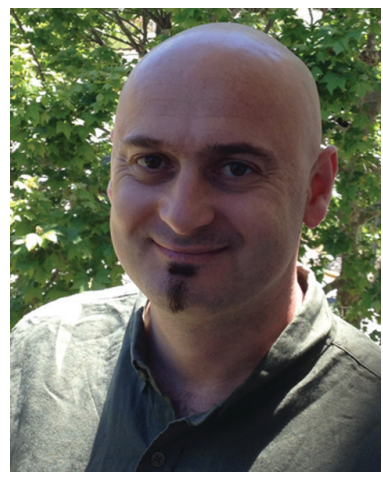

Laurent Commeiras
Laurent Commeiras received his $P h D$ degree in organic chemistry in 2002 from Aix-Marseille University under the guidance of Dr Jean-Luc Parrain. He then joined the group of Prof. Sir Jack E. Baldwin at the University of Oxford. He went back to the University of Aix-Marseille to start his independent career in 2003, first as an associate professor and as a full Professor. His research interests concern the total synthesis of natural and biologically active compounds and the development of new methodologies including the formation of hetero-, poly- and spirocyclic complex structures.

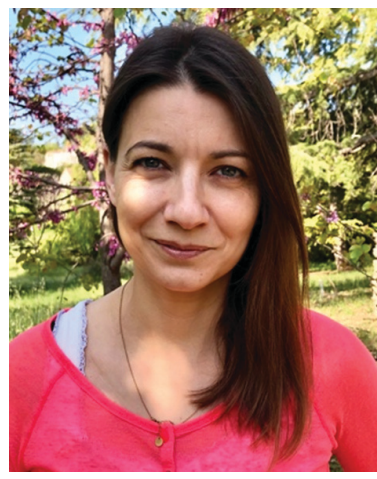

Gaëlle Chouraqui
Gaëlle Chouraqui obtained her $\mathrm{PhD}$ in 2003, from Université Pierre et Marie Curie (Paris France), where she worked under the guidance of Prof. Max Malacria and Dr Corinne Aubert. After a two year-stay as a Postdoctoral Research Fellow with Prof. James H. Rigby at Wayne State University, she returned to Europe and completed a further year as a Postdoctoral Research Fellow in Prof. Ian Paterson's laboratory at the University of Cambridge. She was appointed CNRS Tenured-Researcher in 2007 at Aix-Marseille University (France) and her research interests are centered on the development of novel methodologies to reach molecular complexity, donor-acceptor cyclopropanes and NHC-based catalysts. 


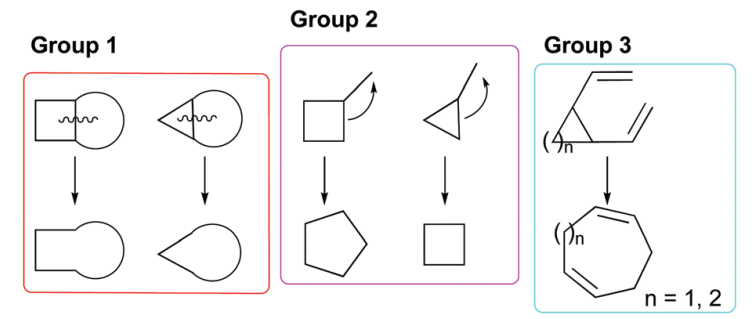

Scheme 1 The three main groups of rearrangements

inventory, but provide the reader with a multifaceted and global vision. We apologise to any author whose paper is not listed here but could be.

\section{Group 1: carbon(s) insertion through opening of a bicyclic system}

This first part is based on solid and well-established methodologies such as fragmentation, retro-aldol, de Mayo, or 1,2elimination reactions. Accordingly, the resulting ring cleavage of fused bicyclic scaffolds depicted here, and containing threeor four-carbon atom cycles, leads to the corresponding allcarbon four- to twelve-membered ones. These recognised transformations ${ }^{11}$ are still relevant since many of them are used for the synthesis of natural product skeletons, therefore showing the indisputable synthetic potential of this kind of strategy. Herein, we decided to focus on contributions about the synthesis of natural molecules and privileged structure motifs found in natural products and/or biologically relevant targets. The different articles have been classified according to the resulting ring-size and we chose to first highlight the challenging task of performing a total synthesis of natural products.

In 2016, Fox and co-workers reported the first enantioselective total synthesis of piperarborenine B (1) in ten steps, with an $8 \%$ overall yield and $92 \%$ ee (Scheme 2$).{ }^{12}$ The key step involved a one pot $\mathrm{Rh}(\mathrm{II})$-catalysed enantioselective bicyclobutanation $^{13} / \mathrm{Cu}(\mathrm{I})$-catalysed homoconjugate addition of $\alpha$-cynnamyl- $\alpha$-diazoesters sequence. The enantioselective decomposition of the 2-diazo-5-arylpent-4-enoate 2 into the corresponding bicyclo[1.1.0]butane 3 at $-78{ }^{\circ} \mathrm{C}$ was followed by a cuprate-mediated ring opening. A subsequent kinetic protonation in presence of a bulky proton source (BHT), finally set the relative stereochemistry on the four-membered ring. The desired diastereomer $\mathbf{4}$ was thus produced in a $4: 1$ ratio. The cyclobutane was obtained in $69 \%$ yield and $92 \%$ ee. The bicyclo[1.1.0]butane ring system is a fascinating moiety since it is probably the most strained bicyclic compound $\left(66.3 \mathrm{kcal} \mathrm{mol}^{-1}\right) \cdot{ }^{14}$ As a result of its highly puckered conformation, both nucleophiles and electrophiles can engage in reaction with bicyclo[1.1.0]butanes. The nucleophilic addition of the Grignard reagent occurs along the axis of the 2p-orbitals forming the central bent $\sigma$-bond, thus explaining the

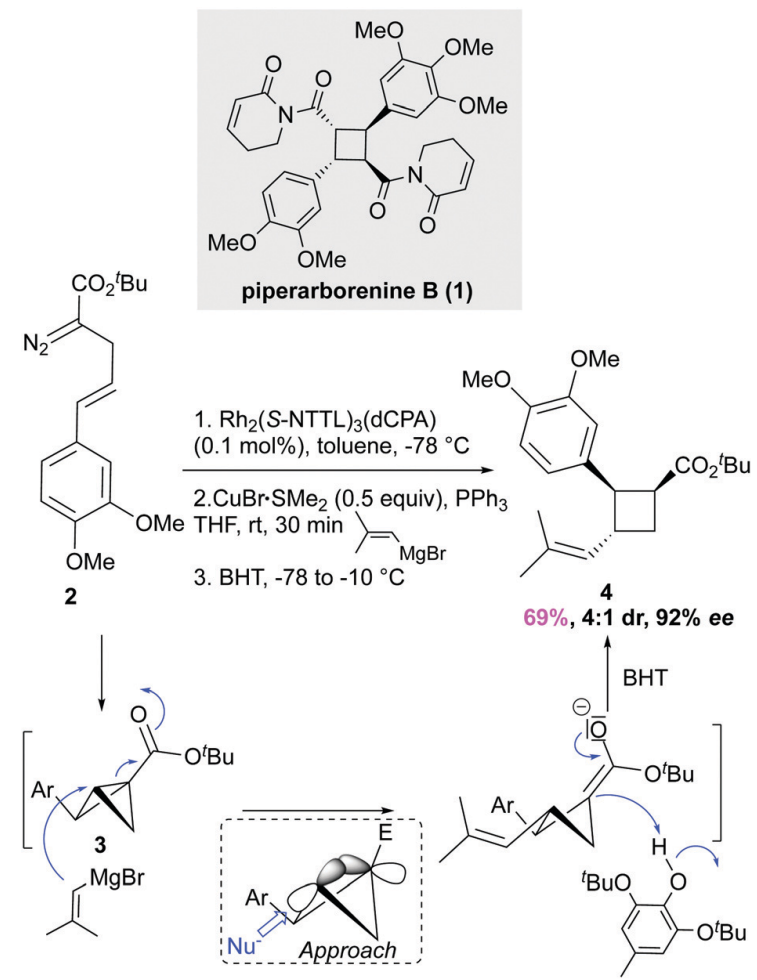

Scheme 2 Approach to piperarborenine B (1) (2016).

relative diastereoselectivity observed (see nucleophilic approach Scheme 2).

Kaurane diterpenoids are integral parts of terpenoid natural products and their attractive structural diversity associated with a wide range of bioactivities have evoked a great deal of interest in the synthetic community. ${ }^{15}$ A regioselective fragmentation of overbred intermediates ${ }^{16}$ was reported by Baran's group for the synthesis of ent-kaurane and beyerane diterpenoids. ${ }^{17}$ Gratifyingly, starting from an embedded polycyclic protected cyclopropanediol derivative 5 and by simple treatment with acyl chloride in methanol at low temperature, the preferential formation of the desired kinetic product 6 was obtained in $79 \%$ (Scheme 3). The undesired thermodynamic adduct 7 was also isolated but in much lower yield 11\% (7.2:1 ratio). Ultimately, this compound set the stage for the final transformation toward the racemic natural product $( \pm)$-steviol (8). The latter was reached in seventeen steps from geranyl acetate in $2.6 \%$ overall yield. The embedded starting structure 5 is the key element enabling the regio- and stereoselective construction of the desired polycyclic core of $( \pm)$-steviol (8).

Very recently, Nelson disclosed an impressive work on the total synthesis of $( \pm)$-vibralactone $(9) .{ }^{18}$ The target was obtained in five steps only ( $4.3 \%$ overall yield). This approach is completely protecting-group-free and the five membered ring is elegantly installed thanks to the fragmentation of a strained [2.1.0] housane structure (Scheme 4). The base-mediated ringexpansion was performed on compound 10, in presence of 1.5 equivalent of DBU in benzene at $75{ }^{\circ} \mathrm{C}$ and for 18 hours. 


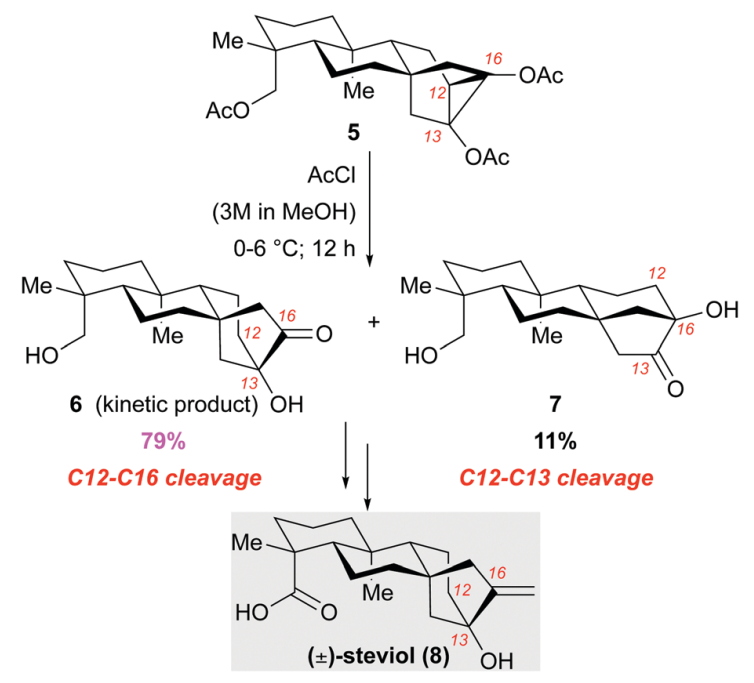

Scheme 3 Approach to $( \pm)$-steviol (8) (2013).

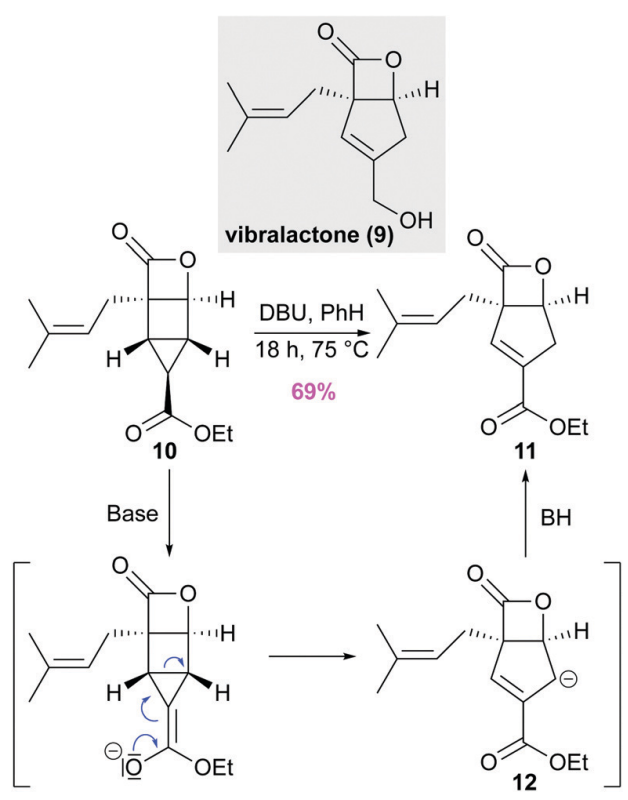

Scheme 4 Approach to ( \pm )-vibralactone (9) (2019)

The regioselective cyclopropane ring cleavage furnished the expected cyclopentene $\mathbf{1 1}$ in $69 \%$ yield, in an atomeconomical fashion. The selectivity of the ring opening was explained by the kinetic protonation of the allyl anion $\mathbf{1 2}$ the furthest away from the sterically congested quaternary centre. Simple reduction of the ester $\mathbf{1 1}$ to the corresponding alcohol in presence of DIBAL-H, gratifyingly delivered the natural product (9). Synthesizing a strained intermediate $\mathbf{1 0}$ was totally worth the effort, since it is the shortest reported synthesis of ( \pm )-vibralactone (9) to date. ${ }^{19}$ Another classical reaction has been utilised to access medium-size carbocyclic systems of natural products on number of occasions. ${ }^{20}$ It relies on the well-known

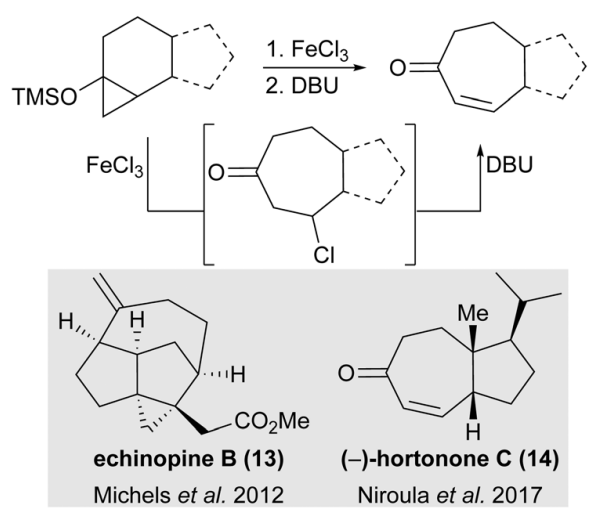

Scheme 5 The Saegusa reaction in the total synthesis of natural products.

Saegusa one-carbon ring expansion protocol ${ }^{21}$ of [4.1.0] bicyclic structures (Scheme 5). Accordingly, the corresponding sevenmembered ring found in the intricated unusual structure of echinopine B (13) $)^{22}$ and the cytotoxic terpenoid (-)-hortonone C $(\mathbf{1 4})^{23}$ was satisfyingly delivered thanks to this approach. Parented examples were also described in the literature and allowed the total synthesis of $( \pm)$-schindilactone A $(\mathbf{1 5})^{24}$ (eqn (1)) and rameswaralide (16) (eqn (2)) (Scheme 6). ${ }^{25}$ Respectively, silver salts were used instead of iron or a radical pathway undertaken, on a necessary brominated cyclopropane derivative. The overall process provided an elegant and facile procedure for the conversion of six-membered rings into the one carbon ring-expanded enones and proved useful in the synthesis of natural products containing such ring systems. These last four examples brought closure to the section devoted to the total synthesis of natural products and it is worth noting that, carbocyclic skeletons from four- to seven-membered rings could be reached. Next, a comprehensive inventory of the different approaches to relevant targets, with interesting structural complexity and as a potential mean to explore the chemical space in drug design is given.

Cyclic scaffolds featuring benzo-fused-(6,7)-bicyclic moieties are structural motifs found in many biologically active natural and unnatural molecules. ${ }^{26}$ Carbon insertion through opening of a bicyclic system is one of the methods allowing the construction of these challenging structures. ${ }^{27}$ These transformations enable the synthesis of molecular scaffolds with welldefined three-dimensional arrangement in a straightforward manner. ${ }^{28}$ For instance, Meng and Cao uncovered a goldand triflic acid co-catalysed [4+2]/ring expansion domino cascade (Scheme 7). ${ }^{29}$ Reaction of ortho-alkynylaldehyde 17 with cyclopropenes 18 in presence of $10 \mathrm{~mol} \%$ gold catalyst and 0.5 equivalent of $\mathrm{TfOH}$ in dioxane at $90{ }^{\circ} \mathrm{C}$, provided an efficient access to the corresponding benzo[7]annulene 19 in good yields, with an excellent diastereoselectivity and a complete $Z$-selectivity of the exocyclic olefin. The proposed mechanism suggested that, a gold-mediated 6-endo-dig cyclisation by the oxygen atom could then be followed by an endo-intermolecular Diels-Alder cycloaddition reaction with the cyclopropene $\mathbf{1 8}$, 

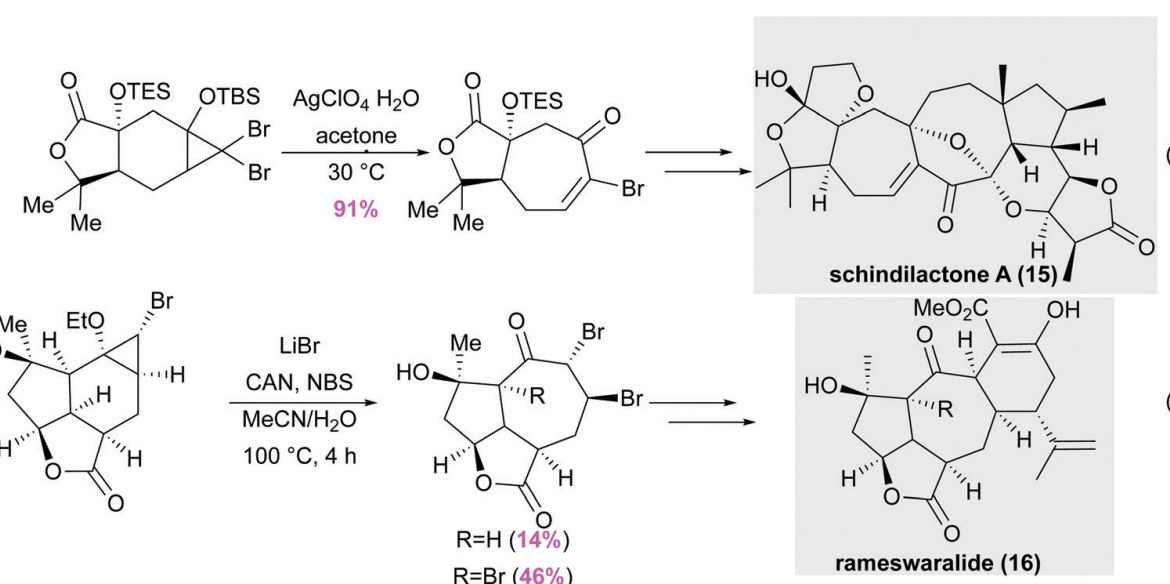

(Eq. 1) Ren et al. 2012
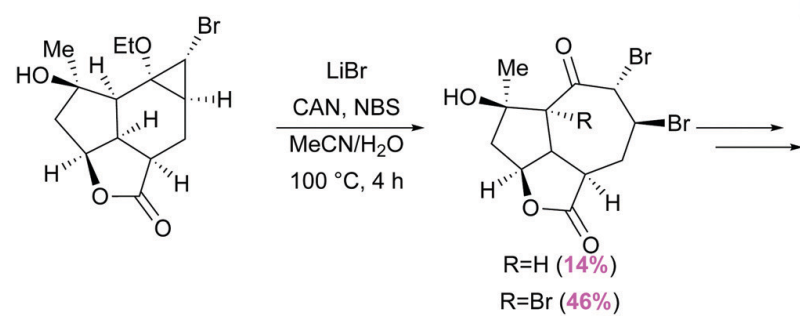

(Eq. 2) Truax et al. 2019

Scheme 6 Approach to schindilactone A (15) and rameswaralide (16)

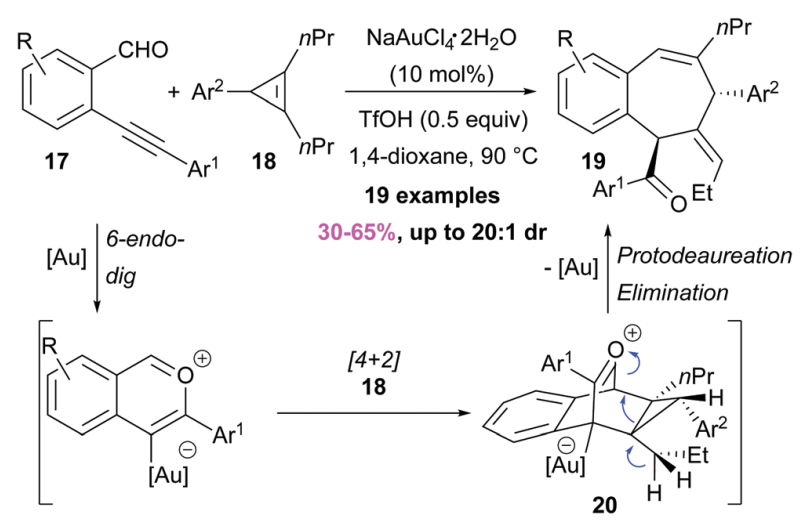

Scheme 7 [4+2]/ring expansion domino cascade (2019).

playing the role of the dienophile. Next a protodeauration on the tricyclic intermediate $\mathbf{2 0}$ and an elimination step, could afford the [4.5.0] bicyclic derivative 19. Due to unfavourable entropic factors and transannular interactions, sevenmembered carbocyclic rings are relatively more difficult to synthesize than their five- or six-counterparts. Consequently, developing strategies to reach this kind of platforms is always needed. On the other hand, bridged ring systems are highly valued as they feature key complexity descriptors ${ }^{30}$ and the imparted ring strain renders their synthesis more difficult than the fused ones.

In 2015, Qu and co-workers ${ }^{31}$ reported an isolated example of bridged ring system construction via the ring expansion of a cyclopropyl bicyclic derivative. The rearrangement of the tricyclic structure 21 occurred in a boiling 9:1 mixture of water and 1,4-dioxane and allowed the construction of the [4.3.1] bicyclic structure 22 albeit as a minor product and therefore in low yield (12\%) (Scheme 8). The major compound 23 came from the non-fused $\mathrm{C}-\mathrm{C}$ cyclopropyl bond cleavage, via the more stable tertiary carbocation, and was isolated in $62 \%$ yield. It is noteworthy to mention that such a strategy, also allowed
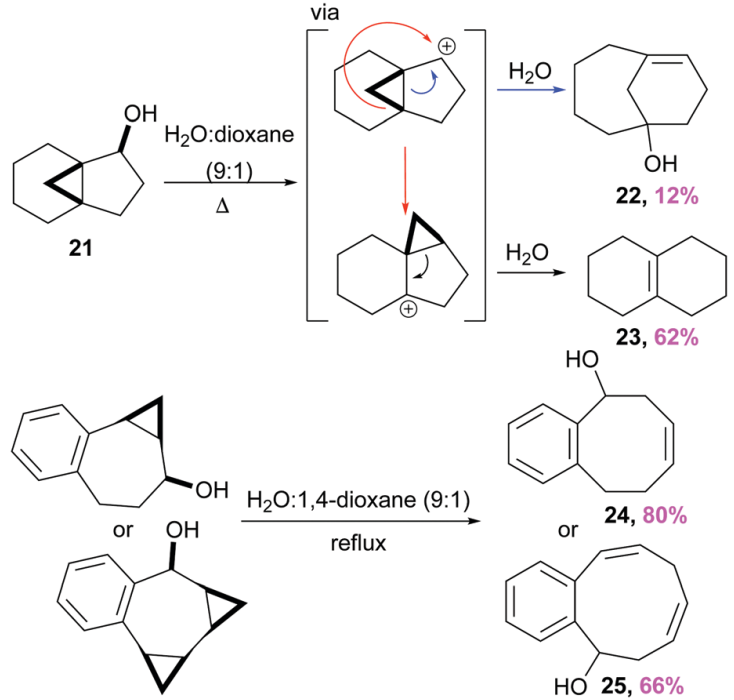

Scheme 8 Construction of bridged ring system 22 (2015).

the formation of fused eight $\mathbf{- 2 4}$ and nine-membered carbocyclic rings 25 , which are even more complicated to obtain than the seven one. The medium-size rings (from eight- to elevenmembered rings) synthesis represent a real synthetic challenge for organic chemists due to the high ring strain. ${ }^{32}$ The release of small ring strain via their expansion, once again, proved beneficial to overcome the unfavourable entropic and enthalpic factors inherent to the formation of higher order cycloadducts.

Gui also studied the cyclopropane scission of tricyclo[4.4.1.0 $\left.0^{1,6}\right]$ undecane, for the unified synthesis of ten cyclocitrinols. ${ }^{33}$ After in-depth studies, the authors established a cascade rearrangement on a derivative of pregnenolone 26, an endogenous steroid hormone (Scheme 9). When the latter was submitted to $m$-CPBA the corresponding sulfoxide was oxidised to sulfone and underwent an elimination step. After the supposed cyclopropyl intermediate 27 formation, the ring expansion would 


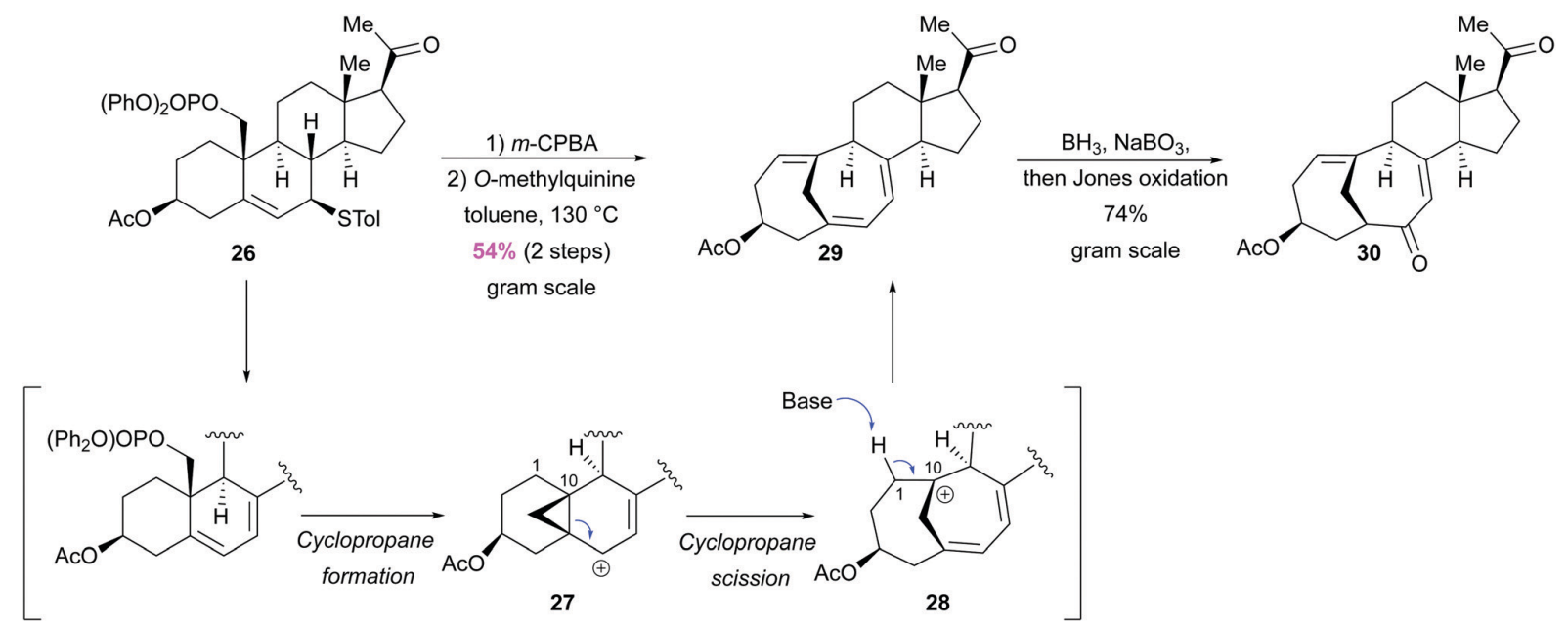

Scheme 9 Approach to a [4.4.1] bicyclic system (2019).

lead to the cycloheptadiene 28. A careful examination of the base used in such process allowed the regioselective installation of the last double bond at the C1-C10 bond. The desired abeo-steroid 29 was obtained on a gram scale in $54 \%$ yield (over two steps) and with $8.2: 1$ regioselectivity. Thereafter, the key intermediate 30 was utilised to reach ten cyclocitrinols in ten to twelve steps from pregnenolone. We already established the difficulty to access bridged ring system, owing to ring strain of this kind of scaffold. Here, the authors added an additional challenge since they even managed to control atropisomerism via point-to-planar chirality transfer.

Another interesting application of the ring expansion of four-membered rings this time, has been reported and allows the formation of cyclohepta[$[b]$ indoles, a structure motif displaying a broad spectrum of biological activities. ${ }^{34}$ Yang and Deng reported a one-pot enantioselective sequence starting with an organocatalysed Michael addition of cyclobutanone 31 onto 2-nitrovinylindole 32 (Scheme 10). ${ }^{35}$ This reaction led to intermediate $\mathbf{3 3}$ and epi-33 which, upon action of boron trifluoride etherate Lewis acid, underwent a cyclisation to the five-membered ring. The latter could spontaneously perform a fragmentation and deliver the corresponding medium-size indolic systems in yields up to $84 \%$, as a mixture of two diastereomers cis- and trans-34 respectively and with excellent enantioselectivities. The stereoselectivities have been rationalised: the diastereoselectivity could originate from the protonation step of the amide enolate and the enantioselectivity from the Michael addition step catalysed by a chiral bifunctional aminocatalyst. The authors not only demonstrated the broad substrate scope and the scalability of the process but also, the high synthetic potential of the obtained imbedded scaffolds.

Another approach to cyclohepta $[b]$ indoles and other useful seven- and even eight-membered carbocyclic platforms, relies on an intramolecular alkyne-de Mayo reaction (Scheme 11). ${ }^{36} \mathrm{~A}$ photochemically induced cascade process starting from 35 in a polar protic solvent provided the desired adduct 36 in excellent yields. The mechanism to the seven-membered ring

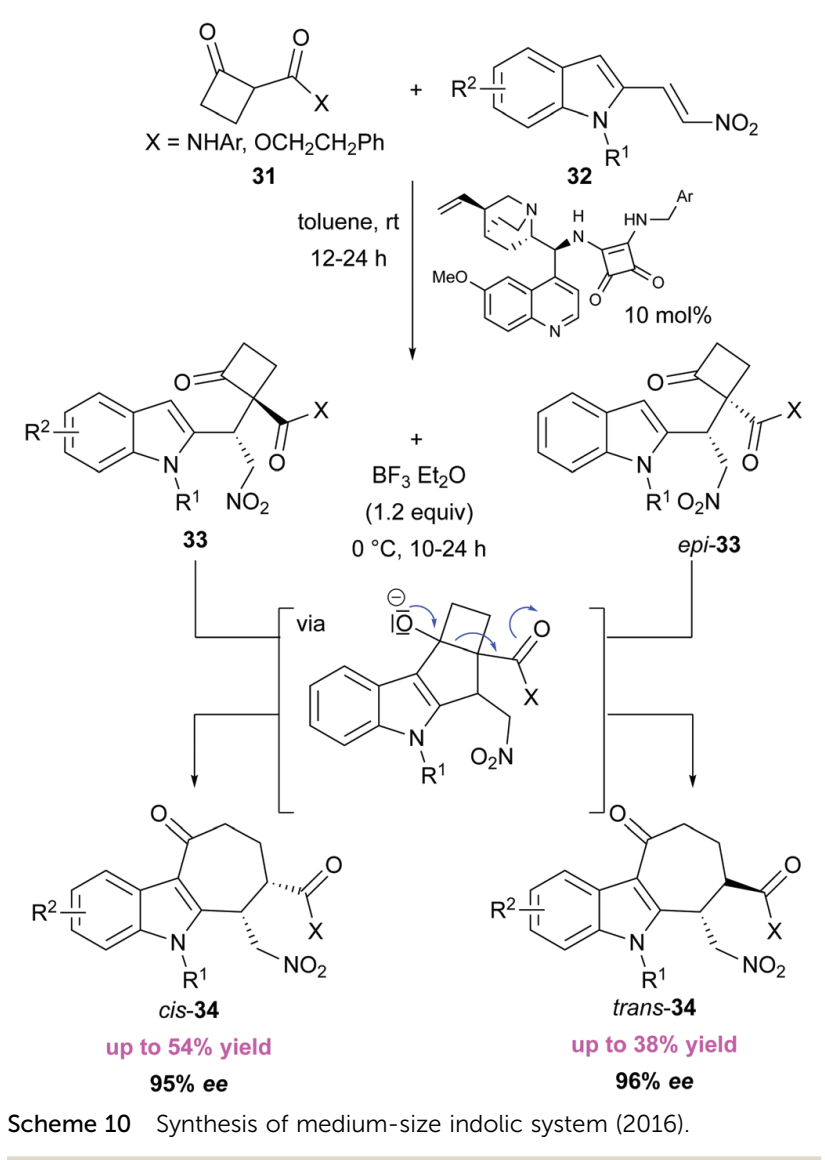

presumably proceeds through the intermediate tricyclic cyclobutene $c i s-S_{0}-37$ via a retro-Mannich reaction.

As already stated, the formation of challenging larger ring systems can benefit from the strain release of small-size cyclic compounds. In 2016, Li and co-workers showed that the 


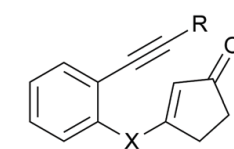

35

$\mathrm{T}_{1}-35$

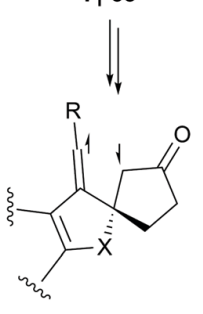<smiles>[R]C#Cc1ccccc1[Y]([Y6])([H])C1=C(CC)CCC1=O</smiles>

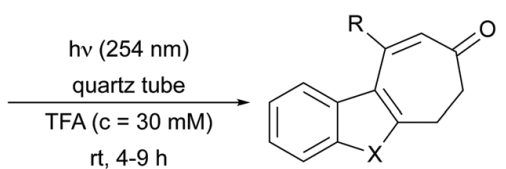

$\mathrm{X}=\mathrm{NBoc}$

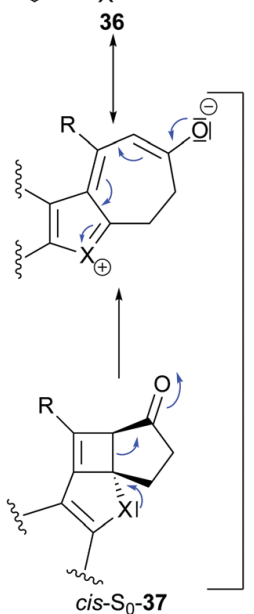

Scheme 11 Approach to cyclohepta[b]indoles (2018)

unstrained system 38 , strictly under basic conditions, in a metal-free medium, and in presence of a cyclic keto ester 39, led to the corresponding eight-membered ring $\mathbf{4 0}$ in excellent yields (Scheme 12). ${ }^{37}$ This transformation tolerated a range of different substituents both on the alkyne and/or the aromatic part of the molecule. A closer look at the suggested mechanism is necessary to understand why this example is part of this review. Michael addition of the enolate of 39 onto the keto alkyne 38 provided intermediate $\mathbf{4 1}$. The latter could undergo an intramolecular aldol reaction leading to the strained

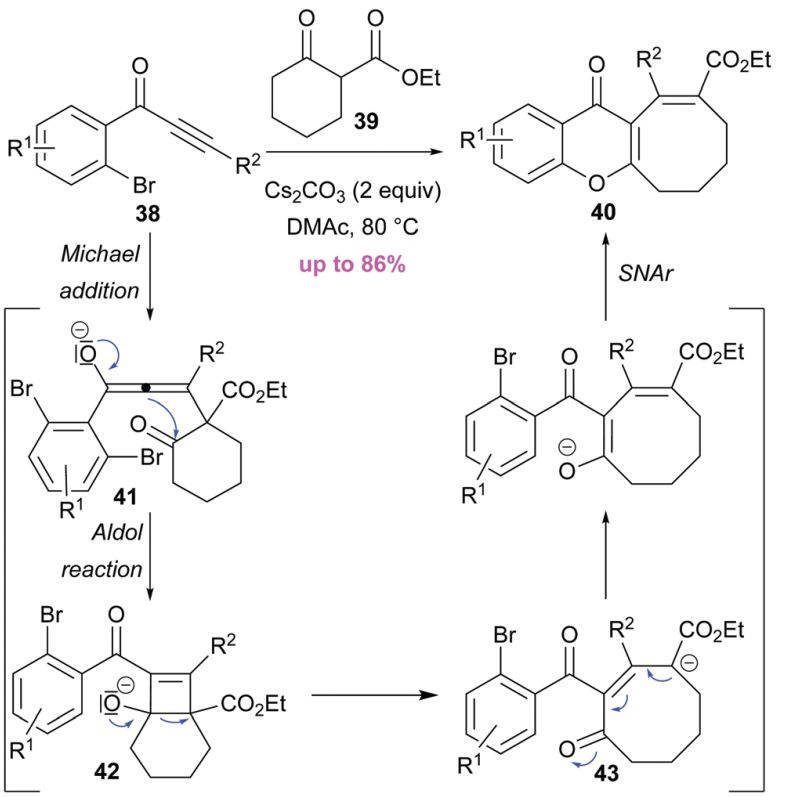

Scheme 12 Synthesis of cycloocta[b]chromene (2016).

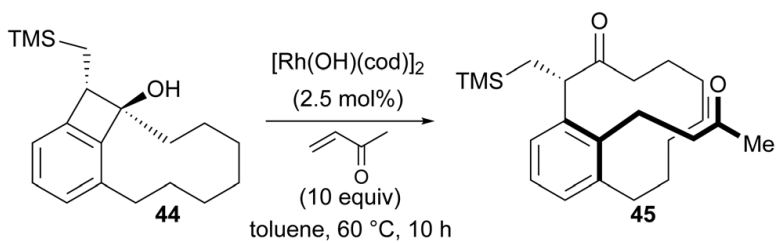

Scheme 13 Stereospecific construction of metacyclophanes (2014).

four-membered ring $\mathbf{4 2}$, which could spontaneously evolve to the desired all-carbon eight membered cycle 43. Overall, this protocol allowed an internal alkyne insertion into a $\mathrm{C}-\mathrm{C}-\sigma$-bond of cyclic $\beta$-dicarbonyl compounds, starting from easily available starting materials and under mild reaction conditions. A new synthesis of cycloocta $[b]$ chromene was thus delivered. ${ }^{38}$

Finally, an example stood out and was reported by Murakami. ${ }^{39}$ The stereospecific construction of metacyclophanes with planar chirality was possible thanks to a two steps sequence starting from orthocyclophanes with central chirality. Cyclophanes are strained molecules playing an important role in supramolecular assembly, and that are also found in natural products and in some pharmaceuticals. ${ }^{40}$ Consequently, stereoselective method to access such skeleton is of paramount importance. Exposure of benzocyclobutanol 44 to $2.5 \mathrm{~mol} \%$ of rhodium catalyst in toluene at room temperature, in presence of a Michael acceptor (10 equiv.) furnished the ring expanded product 45 in $82 \%$ yield with central-to-planar chirality transfer $(>20: 1)$ (Scheme 13).

The few examples presented above showed that the design and construction of building blocks, as integral part of natural products and/or biologically relevant structures, are still an important area of research. Total synthesis continues to be an exciting and inspirational field for organic chemists. ${ }^{41}$ Potent methodologies based on strain-release driven transformations, were reported here, and allowed an access to complex molecular structures containing, for instance, bridged systems, fourmembered and medium-size rings.

\section{Group 2: 1,2 atom migration}

The 1,2-alkyl migration of one of the $\mathrm{C}-\mathrm{C}$ bonds of small cyclic systems onto an adjacent electrophilic carbon centre, is another general method for the ring expansion to the corresponding four- and five-carbocyclic compounds. The driving force of this 1,2-atom migration usually comes from the relief of angle strain, torsional strain or steric crowding and, is driven by the presence of a tertiary alcohol (or an ether or even an amine) therefore generating a carbonyl group. Semipinacol and Tiffeneau-Demjanov methods meet these criteria and, to some extent, Wagner-Meerwein rearrangement can be associated to this kind of mechanism (Scheme 14).

\subsection{Semipinacol rearrangement}

The ring expansion of cyclopropanols and cyclobutanols have been repeatedly utilised in a well-known classical reaction, the 


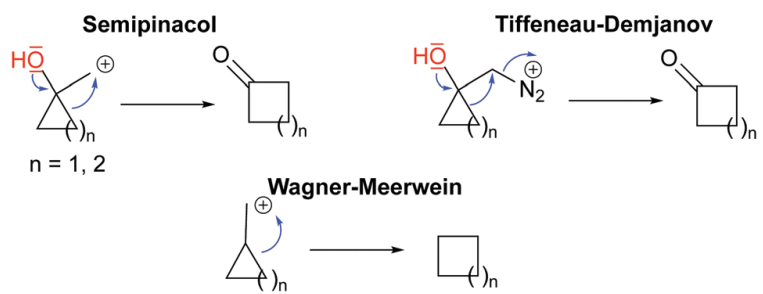

Scheme 14 1,2-Atom migration and ring expansion reactions.

semipinacol rearrangement. ${ }^{42}$ This transformation takes advantage of the electron pushing effect of the hydroxyl group. Accordingly, last year, Natho et al. ${ }^{10 a}$ gave an account on the recent progress made on the ring expansion of cyclobutanols. Le Bras and Muzart, ${ }^{10 b}$ for their part, focused on the Pdcatalysed reactions of cyclopropanols and cyclobutanols. In 2019, Dai enumerated the different total syntheses using a cyclopropanol pathway. ${ }^{9}$ Obviously, the development of scaffolds bearing these privileged moieties (cyclopropanols ${ }^{43}$ and cyclobutanols) is still a topic of importance. The semipinacol rearrangement of the aforementioned species has therefore been quite covered and we refer the reader to the different reviews for further references. Consequently, this part is intended to provide an update overview for the transposition of cyclopropanols (since 2012) and to highlight the rearrangement of alkenyls cyclobutanols since 2019.

3.1.1. Semipinacol rearrangement of cyclopropanols. Goldcatalysed approaches are important for the semipinacol rearrangement of cyclopropanols. ${ }^{44}$ This strategy gives access to cyclobutanes, a building block not only frequently present in natural products, ${ }^{45}$ but also a precursor to more complex structures through ringopening, ring-expansion, and ring-contraction reactions. ${ }^{4,7,10}$
The cycloisomerisation of cyclopropylenynes and analogues initially developed by Echavarren and Toste, ${ }^{46}$ has found application for instance, in the synthesis of the protoilludane core of russujaponol D (46) (Scheme 15). ${ }^{47}$ When allenene 47 was submitted to a cationic gold catalyst, the corresponding $(5,6,4)$ tricyclic structure 48 was obtained in $92 \%$ yield as a mixture of two regioisomers $(2: 148 \mathbf{4}: 48 b)$. From a mechanistic point of view, this reaction could proceed through a 5-exo-trig cyclisation, triggered by a prior coordination of the gold catalyst to the allene 47 . The carbocationic intermediate $\mathbf{4 9}$ could then undergo the semipinacol rearrangement to the four-membered ring 50. A Prins cyclisation, followed by an elimination and a subsequent protodemetallation could afford the observed tricyclic structures $\mathbf{4 8 a}$ and $\mathbf{4 8 b}$. Another example relied on the enantioselective version of the Au-mediated rearrangement of cyclopropylenynes. ${ }^{48}$ In presence of a chiral bis(phosphine)-digold(I)catalyst, the corresponding cyclobutanones $\mathbf{5 1}$ were obtained in yields up to $94 \%$, as a mixture of cis and trans diastereomers (from 1.3:1 to >40:1 cis:trans ratio) and high enantioselectivities (up to $99 \%$ ee) (Scheme 16).

Pioneer work from Toste ${ }^{49}$ has also shown that, alkynylcyclopropanols could be employed in gold-mediated ring expansion, thus delivering alkylidenecyclobutanones. More recently, an enyne system has been tethered by a cyclopropane ring $\mathbf{5 2}$ and the gold-catalysed ring expansion provided, in this case, a tetrasubstituted methylenecyclobutanone 53 (Scheme 17). ${ }^{50}$ The proposed mechanism has been supported by a range of experiments, as well as DFT calculations. The reaction proceeds through a ring expansion induced by a gold-complexation to the alkyne 54. Intermediate 55 then isomerises to carbenoid 56. The ensuing [3,3]-sigmatropic rearrangement affords the cyclobutanone 57, which then undergoes a $[1,2]$-alkyl shift in presence of a catalytic amount of water.

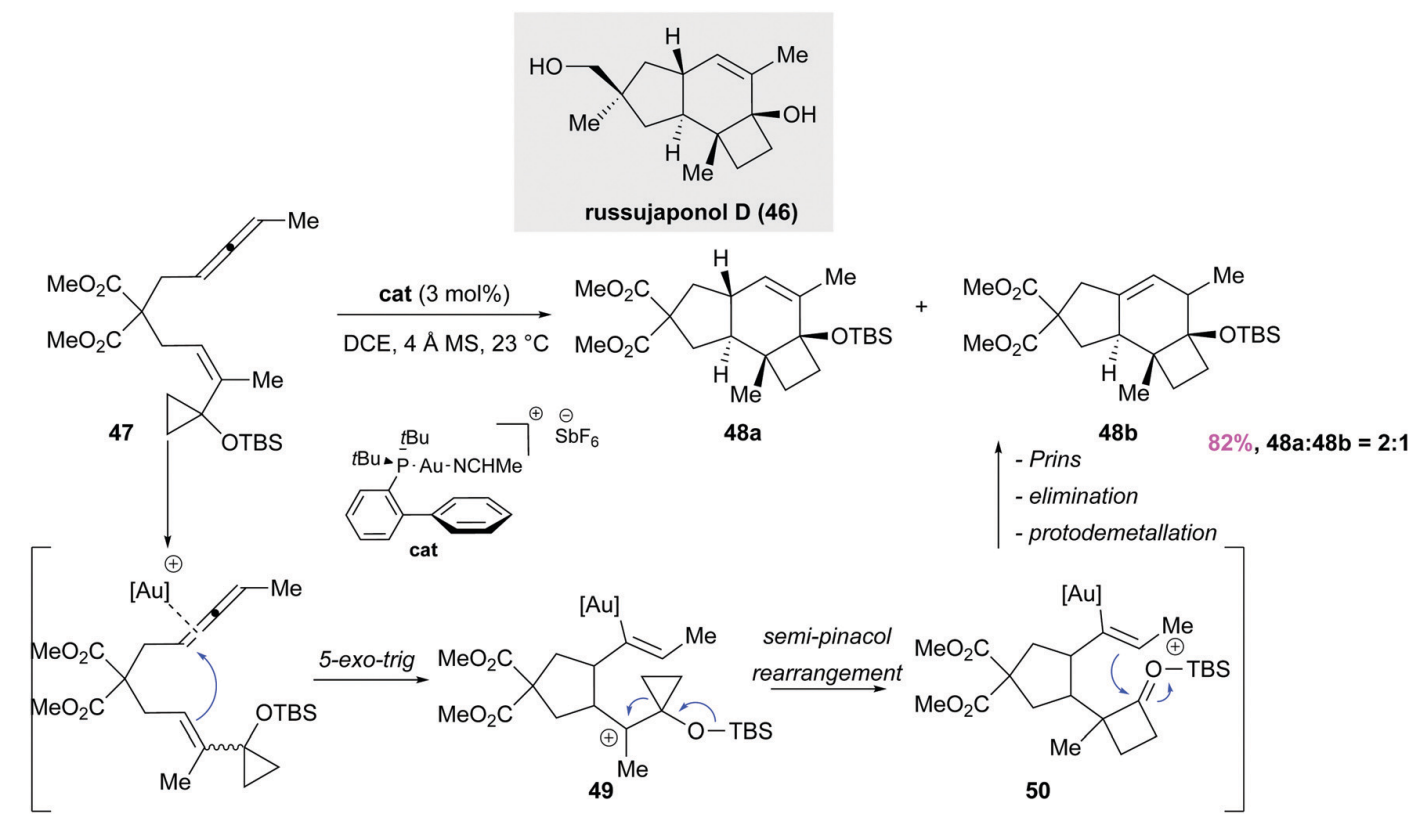

Scheme 15 Approach to russujaponol D (46) (2013). 


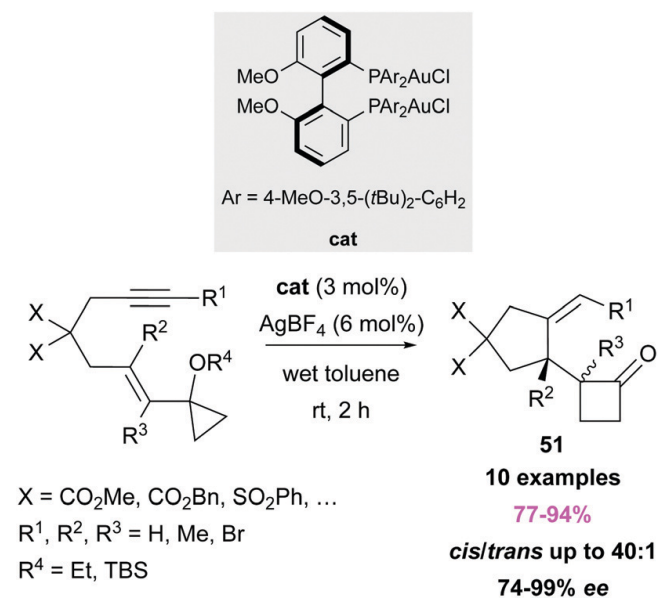

Scheme 16 Stereoselective rearrangement of cyclopropylenynes (2017).

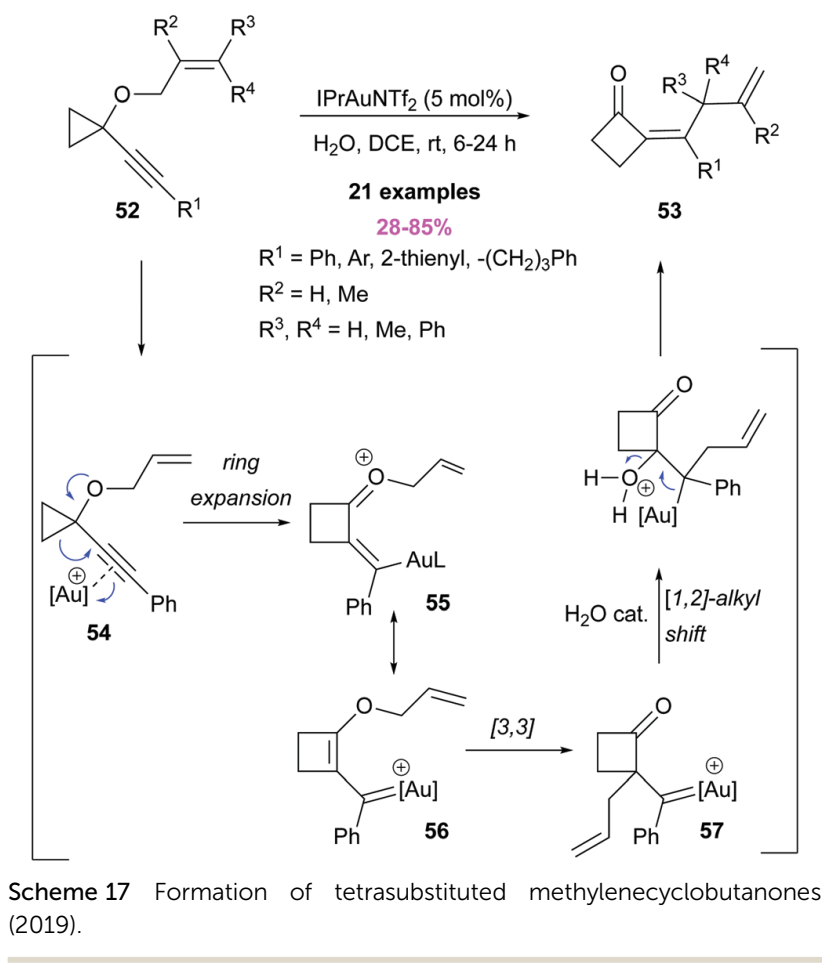

Finally, an intermolecular gold-mediated reaction between 1,6enynes 58 and a cyclopropene derivative $\mathbf{5 9}$ has been envisioned by Matsuda et al. (Scheme 18). ${ }^{51}$ Mechanistically, the cycloisomerisation to 60 could occur first and the latter could react with the cyclopropenone 59 to generate the corresponding oxocarbenium ion intermediate 61. A stepwise intramolecular [3+2] reaction to cyclopentenone $\mathbf{6 2}$ via intermediate $\mathbf{6 3}$ could deliver the spirocyclic structure 64. This last example is related to a semipinacol rearrangement but cannot be classified as such since the ring expansion occurred via a 1,3-alkyl shift. However, it was worth underlining this ring-expansion of small-size ring leading to the synthetically challenging spiro structure.

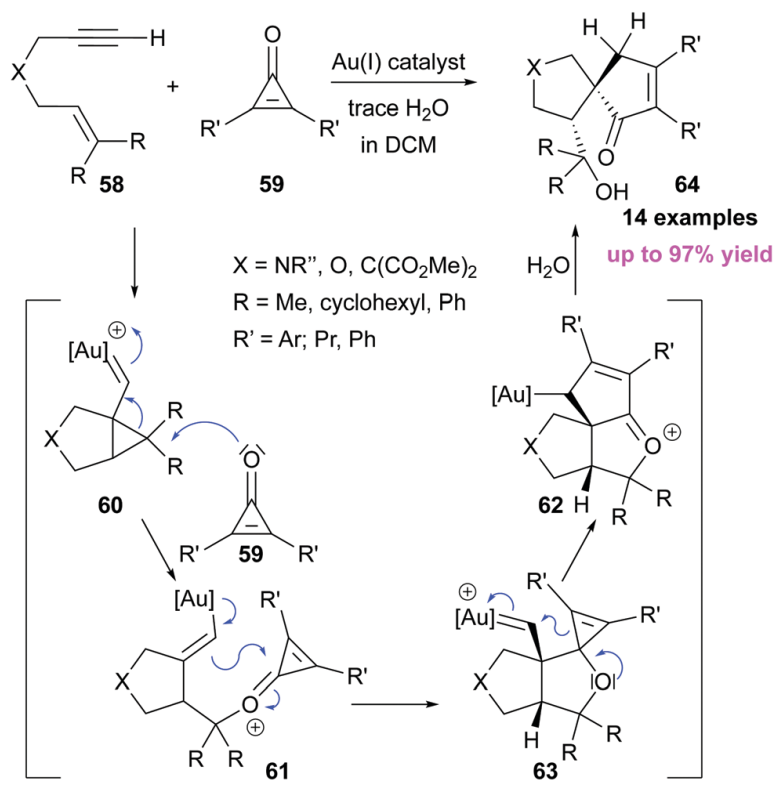

Scheme 18 Stereoselective rearrangement of cyclopropylenynes (2014).

Photoredox catalysis is another mean to achieve semipinacol rearrangements. Accordingly, Sahoo et al. reported in 2015, the visible-light induced $\beta$-trifluoromethylation of cyclopentanones starting from the corresponding alkenylcyclobutanols (see previous section for an ionic example of trifluoromethylation/ semipinacol rearrangement cascade). ${ }^{52}$ On the other hand, a $\mathrm{N}$-centred radical 65 generated via visible-light photocatalysis was reported by Liu et al. and reacted on an alkylidene cyclopropane $\mathbf{6 6}$ as a precursor to the transient cyclopropanamine cationic species 67 (Scheme 19). ${ }^{53}$ The latter could then undergo an aza-semipinacol rearrangement to the cyclobutanimine 68. This strained derivative was thereafter utilised, for the synthesis of butyrolactone 69 exhibiting a quaternary carbon centre, therefore demonstrating the synthetic power of the described method.

Finally, we wanted to end this section with a transition metal-free domino reaction. In presence of a chiral Lewis acid catalyst, $\alpha$-silyloxyacroleins 70 and donor-acceptor diazo derivatives $\mathbf{7 1}$ underwent a one-pot cyclopropanation/semipinacol rearrangement cascade reaction (Scheme 20). ${ }^{54}$ The chiral oxazaborolidinium ion catalyst (cat) delivered the corresponding $\alpha, \beta$-substituted-cyclobutanones 72 (including a $\beta$-quaternary centre) in high yields (up to $91 \%$ ) and with excellent enantio- and diastereoselectivity (up to $98 \%$ ee and up to $>20: 1 \mathrm{dr}$ ). Derivatisation of cyclobutanones to various cyclic building blocks ( $\gamma$-lactones, cyclobutanols, cyclopentanones) highlighted the synthetic potential of the method.

3.1.2. Semipinacol rearrangement of alkenyl cyclobutanols. The semipinacol rearrangement of alkenyl cyclobutanols has been the focus of great attention until to date and has allowed the concomitant formation of the corresponding cyclopentanones with the challenging installation of an $\alpha$-quaternary stereocentre. ${ }^{42,55,56}$ In the literature, two types of 

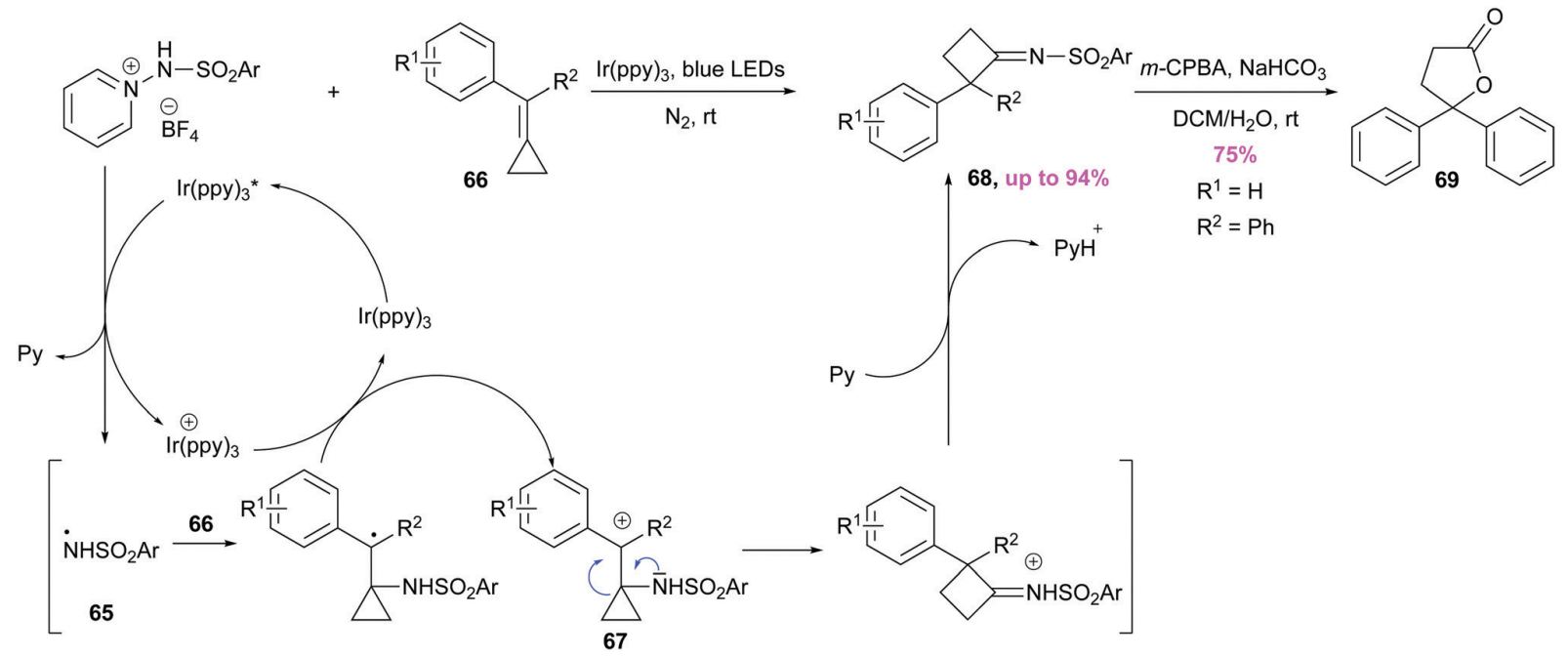

Scheme 19 Visible-light-induced aza-pinacol rearrangement (2017).

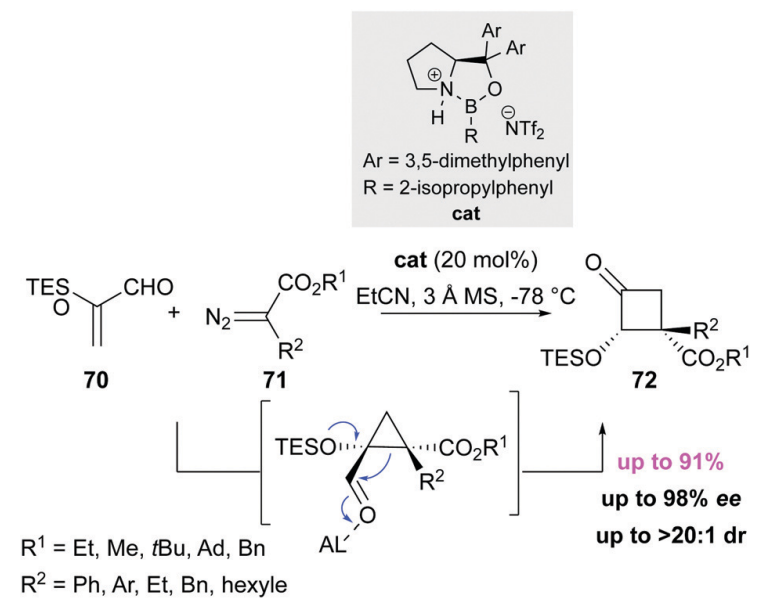

Scheme 20 A cyclopropanation/semipinacol rearrangement cascade reaction (2018).

mechanisms of the domino functionalisation/rearrangement of cyclobutanols to cyclopentanones has been identified: either electrophile-induced or radical-initiated rearrangements. ${ }^{10}$ The idea is to develop a full or latent cationic charge $\alpha$ to the tertiary

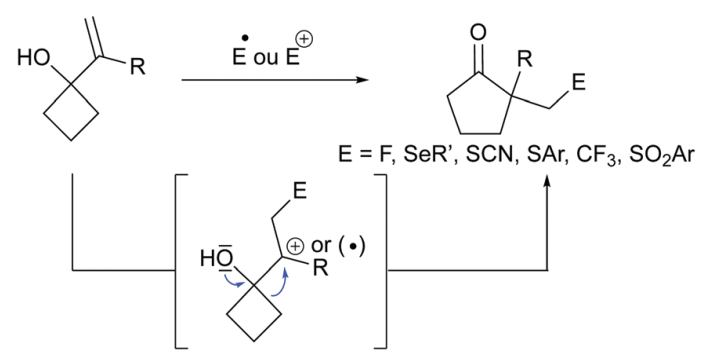

Scheme 21 Recent approaches to $\alpha$-substituted cyclopentanones (2019). alcoholic carbon, to trigger the ring expansion. Recent advances reported the installation of $\alpha$-fluoromethyl, ${ }^{57}$ $\beta$-selenyl, ${ }^{58} \beta$-thiocyano, ${ }^{59} \beta$-sulfenyl, ${ }^{60} \beta$-trifluoromethyl ${ }^{61}$ and $\beta$-sulfonyl ${ }^{61,62}$ cyclopentanones (Scheme 21). Compared to previous strategies, these metal-free approaches advantageously occurred under mild conditions, were often environmentally benign and provided good yields of the desired products. For instance, Chen and Zhang described the first electrochemical trifluoromethylation and sulfonylation/semipinacol rearrangements of allylic alcohols using readily available $\mathrm{R}^{\prime} \mathrm{SO}_{2} \mathrm{Na}$ $\left(\mathrm{R}^{\prime}=\mathrm{CF}_{3}, \mathrm{Ph}\right) \cdot{ }^{61}$

Despite important breakthroughs in the field of catalytic asymmetric pinacol rearrangements, ${ }^{63}$ within these recent examples, ${ }^{57-61}$ sole two of them were developed in their enantiomeric versions and were worth underlining here. In the Lewis acid-mediated thiocyano rearrangement of alkenyl cyclobutanols $73,{ }^{59}$ preliminary studies have shown that chiral BINAM-derived selenide $(S)-74$ catalyst, ${ }^{64}$ in presence of $\mathrm{MsOH}$ at $-10{ }^{\circ} \mathrm{C}$, could furnish $65 \%$ of expected adduct 75 with an encouraging $19 \%$ enantiomeric excess, via intermediate 76 (Scheme 22).

On the other hand, enantiomeric excess up to $98 \%$ have been noted in the Lewis base/Brønsted acid co-catalysed sulfenylation/semipinacol rearrangement of di-77 and even trisubstituted 78 allylic alcohols (Scheme 23). ${ }^{60}$ This stereoselective installation of a sulfur substituent proved later on useful in the total synthesis of (-)-herbertene (79). Moreover, such a strategy allowed the installation of two contiguous stereocentres (one being a quaternary one). Obviously, organosulfur derivatives represent attractive features. They are not only required in the field of bioactive natural products and medicines, but also in the design of chiral catalysts/ligands as well as material science.

Still in the framework of developing stereoselective metal-free ring expansions of alkenyl cyclobutanols, Zhang and $\mathrm{Tu}^{65}$ utilised an organocatalytic enantioselective $\alpha$-alkylation/semipinacol 


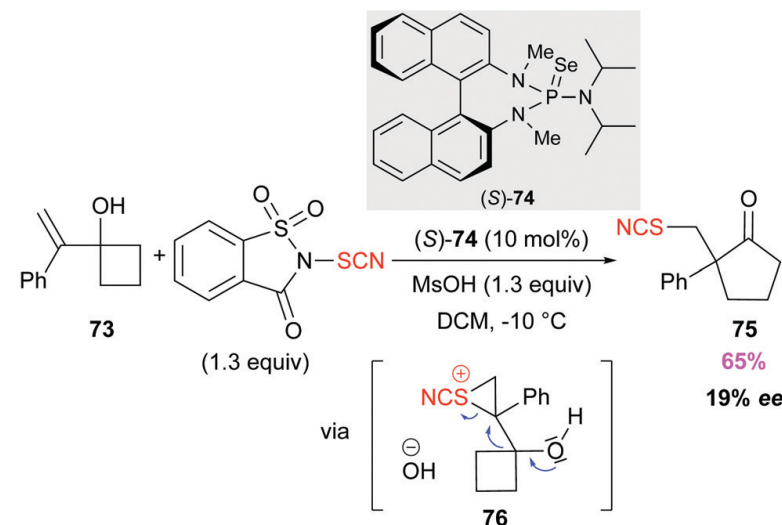

Scheme 22 Catalytic asymmetric thiocyano semipinacol rearrangement (2019).
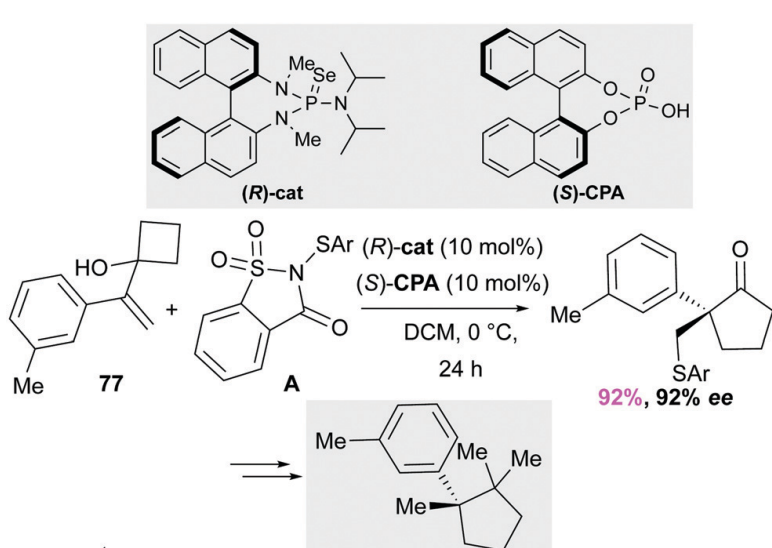

$\%, 92 \%$ ee

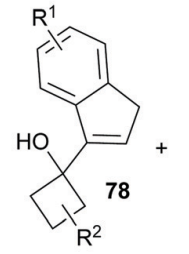

$\mathrm{R}^{1}=\mathrm{Me}, \mathrm{OMe}, \mathrm{Ph}$, etc $\mathrm{R}^{2}=\mathrm{H}, \mathrm{Alk}, \mathrm{Ph}$ (-)-herbertene (79)

(R)-cat $(10 \mathrm{~mol} \%)$ $\mathrm{DCM},-60$ or $-10^{\circ} \mathrm{C}$

$12-24 \mathrm{~h}$

$\mathrm{Ar}=\mathrm{Ph}, 2-\mathrm{Me}-\mathrm{C}_{6} \mathrm{H}_{4}$

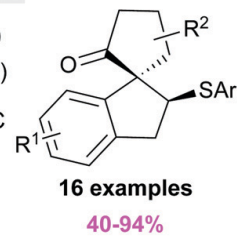

$78-98 \%$ ee (S)-CPA (10 mol\%)

Scheme 23 Asymmetric sulfenylation/semipinacol rearrangement (2019).

rearrangement initiated by an enamine radical cation (Scheme 24). The organo-SOMO catalysed sequence between an allylic cyclobutanol $\mathbf{8 0}$ and an aldehyde $\mathbf{8 1}$ led to the formation of the $\mathrm{C}-\mathrm{C}$ bond between the two partners with concomitant creation of the first stereocentre. Under oxidative conditions, the cyclobutanol 82 underwent a 1,2-alkyl migration onto the adjacent electrophilic carbon therefore delivering the $\alpha$-quaternary- $\delta$ carbonyl cycloketones 83 in good yields, excellent diastereoselectivities and very high enantioselectivities. An intramolecular version of this strategy successfully furnished the (+)-cerapicol (84) natural product.

The same authors reported an elegant total synthesis of lycojaponicumin A (85), a fawcettimine-type alkaloid using,

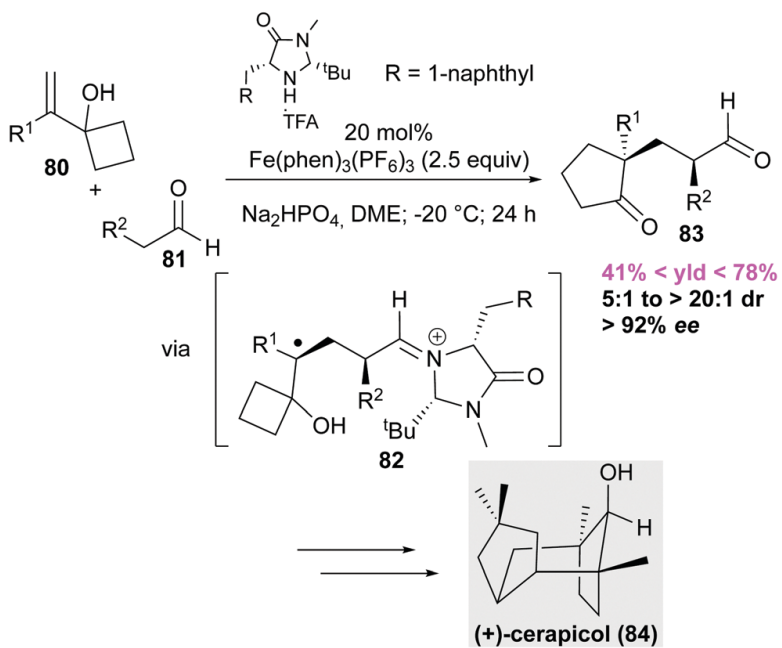

Scheme 24 Asymmetric $\alpha$-alkylation/semipinacol rearrangement (2020).

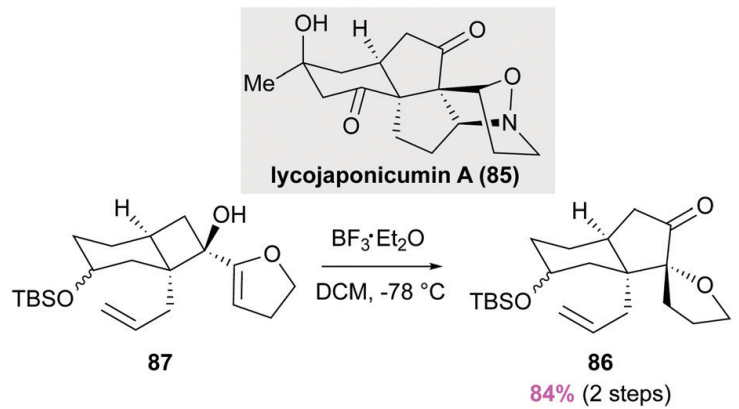

Scheme 25 Key step of the total synthesis of lycojaponicum A (86) (2020)

once again, the semipinacol ring expansion as a key step to reach the $\mathrm{AB}$ core of the natural product (Scheme 25). The challenged spiro derivative $\mathbf{8 6}$ was formed in $84 \%$ yield (over 2 steps), starting from the corresponding cyclobutanol 87 and, by simple treatment with a Lewis acid. ${ }^{66}$ In the following paragraph, we wish to point out that recent progresses have mostly been devoted to semipinacol rearrangements of cyclobutanols as integral part of domino reactions for the synthesis of complex polycyclic skeletons. ${ }^{67}$ Simple ring systems were readily available starting points to create synthetically daunting structural motifs such as intricated polycyclic structures and congested quaternary carbon centres. For example, a catalytic asymmetric one-pot [3+2] cyclisation/semipinacol rearrangement sequence gave birth to multi-substituted $3 H$-spiro[benzofuran-2,2'-cyclopentane] skeletons 88 (21 examples, up to $67 \%$ yields, up to $92 \%$ ee and up to $>20: 1 \mathrm{dr}$ ) (Scheme 26). ${ }^{68}$

Another sequential cascade reaction featured a semipinacol rearrangement/Michael addition/Henry reaction and allowed the formation of $(6,5,5)$-fused tricyclic backbones 89, presenting five contiguous stereocentres, including a quaternary carbon centre and a tetrasubstituted one (Scheme 27). ${ }^{69}$ This step-, 

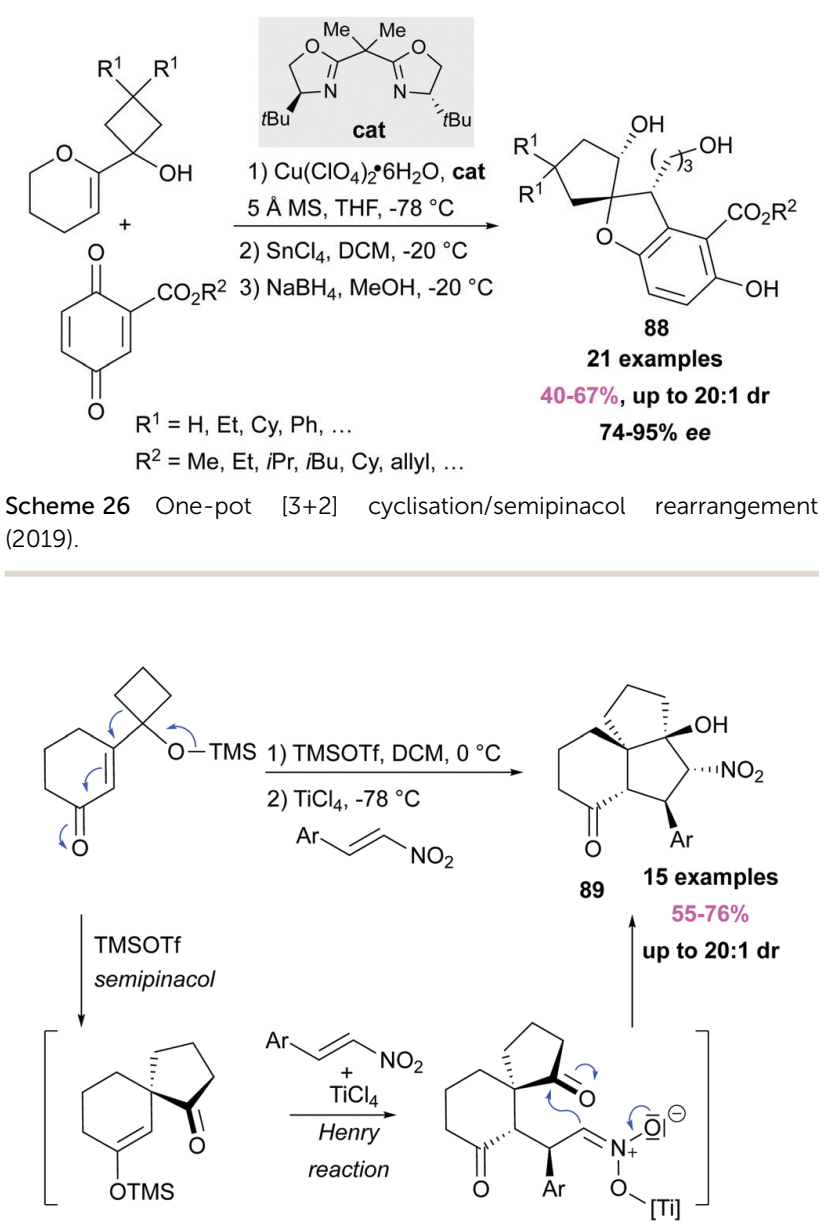

Scheme 27 Semipinacol rearrangement/Michael addition/Henry reaction (2020).

atom-economic transformation was mediated by simple Lewis acids, namely TMSOTf and $\mathrm{TiCl}_{4}$, and provides high diastereoselectivities with a broad substrate scope.

To conclude, alkenyl cyclobutanols are excellent partners to produce cyclopentanones via [1,2]-alkyl shift triggered by a vicinal unsaturation. The recent progress put emphasis on metal-free and, preferably, stereoselective reactions. Additionally, to explore the synthetic viability of the method, the installation of an $\alpha$-quaternary stereocentre was utilised as part of domino reactions in the total synthesis of natural products.

In a similar fashion, the Tiffeneau-Demjanov reaction and the Wagner-Meerwein [1,2]-alkyl shift represent just alternative to the more common semipinacol rearrangement. The Tiffeneau-Demjanov reaction in presence of a diazo derivative, allows the ring extension of cyclopropanones and cyclobutanones to the corresponding cyclobutanones and cyclopentanones respectively, in high yields and selectivities. The less substituted carbon usually migrates with retention of configuration. ${ }^{70}$ Excellent achievements have been reported for the asymmetric version of the reaction and the described systems delivered high levels of enantioselective induction. ${ }^{71}$
Despite these interesting features only few examples were found in the recent literature. ${ }^{72}$ It seems like these kinds of transformations had their finest hours in the late nineties and early years of this century. For those reasons, this topic shall not be addressed in this report. Nowadays Tiffeneau-Demjanov ring enlargement could have its rebirth if involved in novel cascade processes and/or as a key step in the synthesis of natural products.

\subsection{Wagner-Meerwein rearrangement}

The metal-mediated Wagner-Meerwein process often involves an initial cycloisomerisation via activation of a vicinal unsaturation followed by the [1,2]-alkyl shift into the cyclopropylmethyl cation (or carbene) thus created. This past decade, two different type of starting materials and an intermediate have been reported to engage in this ring expansion: on one hand, the methylenecyclopropanes (MCPs) and the alkynyl cyclopropanes and, on the other hand, the carbenoid intermediates. Accordingly, this rearrangement is mostly dominated by the metal-mediated ring opening of MCPs and, has been reviewed twice lately. ${ }^{73}$ Consequently, sole the MCP rearrangements since 2019 , will be highlighted here. We advise the reader to these two publications for documentation and discussion.

3.2.1. MCP rearrangements since 2019. Methylenecyclopropanes are useful building blocks for the construction of elaborate structures containing four-membered rings. Being highly strained $\left(38.8 \mathrm{kcal} \mathrm{mol}^{-1}\right)$, they are prone to oxidative addition. Zhao et al. reported the enantioselective construction of strained spiro[2,3]hexanes via a cascade reaction (Scheme 28). ${ }^{74}$ Formation of the iminium ion from the corresponding enal in presence of a secondary electron deficient amine is followed by a Michael addition onto the electronrich MCP 90. Then, a concerted process for the formation of the new cyclopropane unit and, the ring expansion of the cyclopropyl 91 onto the electrophilic adjacent carbon centre, provides the bicyclic spiro system $\mathbf{9 2}$ after hydrolysis of the iminium.

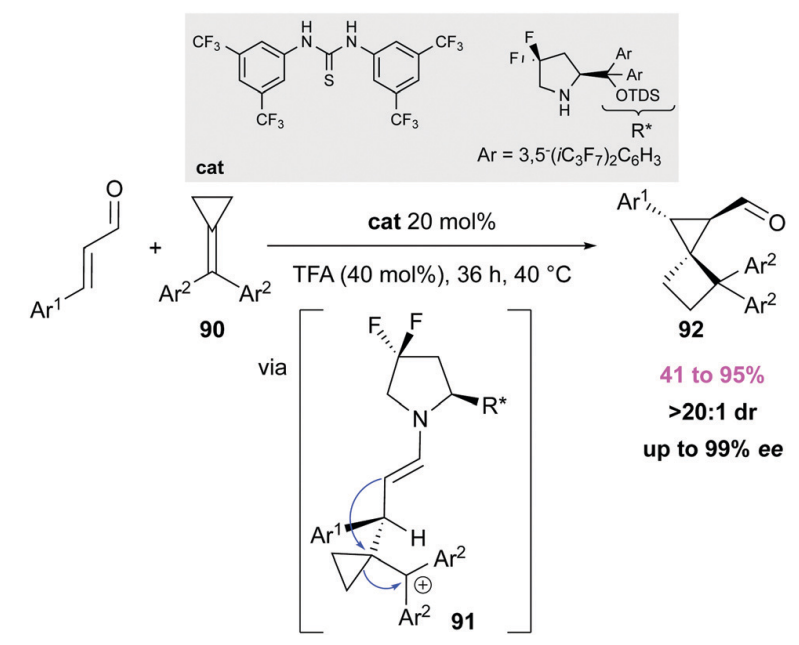

Scheme 28 Construction of strained spiro[2,3]hexanes (2020). 


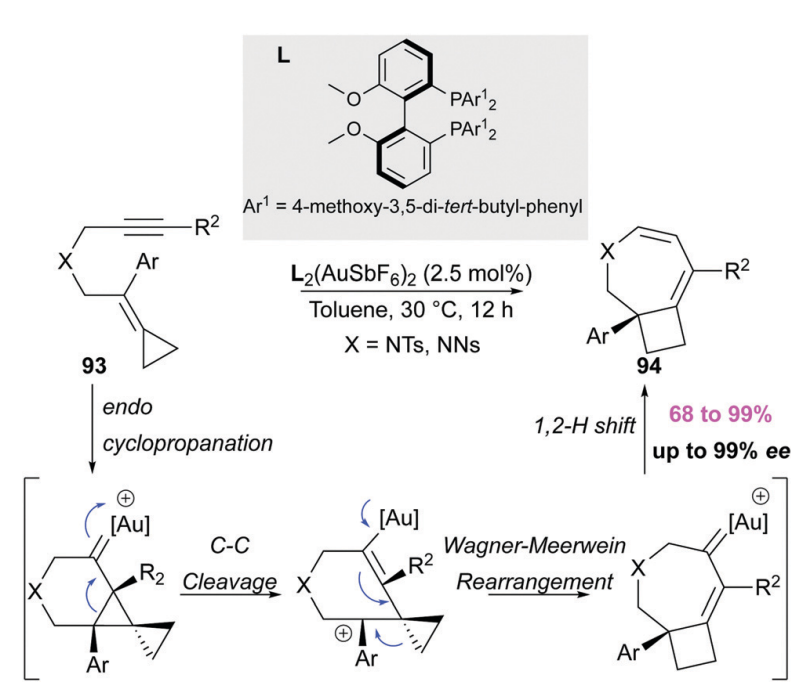

Scheme 29 Stereoselective access to azepine-fused cyclobutanes (2019).

Moderate to excellent yields were obtained with high diastereoand enantioselectivities. This approach represents a highly versatile method giving access to more complex structures that would be difficult if not impossible to synthesise with single cyclisation reactions. This is also an opportunity to explore and expand the chemical space to enable drug discovery.

$\mathrm{Yu}$ employed the rearrangement of MCPs synthetic tool, for a stereoselective access to azepine-fused cyclobutanes (Scheme 29). ${ }^{75}$ When yne-MCP 93 was submitted to a chiral gold catalyst $\mathrm{L}_{2}\left(\mathrm{AuSbF}_{6}\right)_{2}$, a cyclopropanation/C-C bond cleavage/Wagner-Meerwein rearrangement sequence took place. The corresponding 3-azabicyclo[5.2.0]nonadienes 94 were obtained with excellent enantioselectivities. According to DFT calculations, the chirality of the final product would come from the cyclopropanation step via a chirality-memorised WagnerMeerwein rearrangement. Many natural products with significant biological activities have cyclobutane moieties. ${ }^{4 b, 45 a, 76}$ Therefore, efforts to develop methods to synthesise these motifs are highly required so that synthetic chemists could have more tools in their efficient target-, diversity, and function-oriented synthesis. Carreira, demonstrated recently that the yne-MCP rearrangement could be utilised as a key step in the total synthesis of the harziane diterpenoid (95). ${ }^{77}$ The tricyclic fused structure 96 was gratifyingly obtained in $87 \%$ yield, with a $>11: 1 \mathrm{dr}$, thanks to a Au-mediated cycloisomerisation (Scheme 30). Finally, and in a very impressive way, Carreira and Gershoni-Poranne elegantly demonstrated that the MCPs could be involved in a Lewis acid mediated cycloisomerisation (Scheme 31$){ }^{78}$ No late transition metal was required and the polycyclic backbone of natural products 97 , like repraesentin $\mathrm{F},{ }^{79}$ illudiolone ${ }^{80}$ or sterpurene, ${ }^{81}$ could be reached easily and diastereoselectively, via a Prins addition/ring expansion/1,2-silyl shift domino sequence. The presence of the silicon group proved not only useful for the $\beta$-stabilisation of the transient cationic derivative 98, but also served as an entry point for further functionalisation.

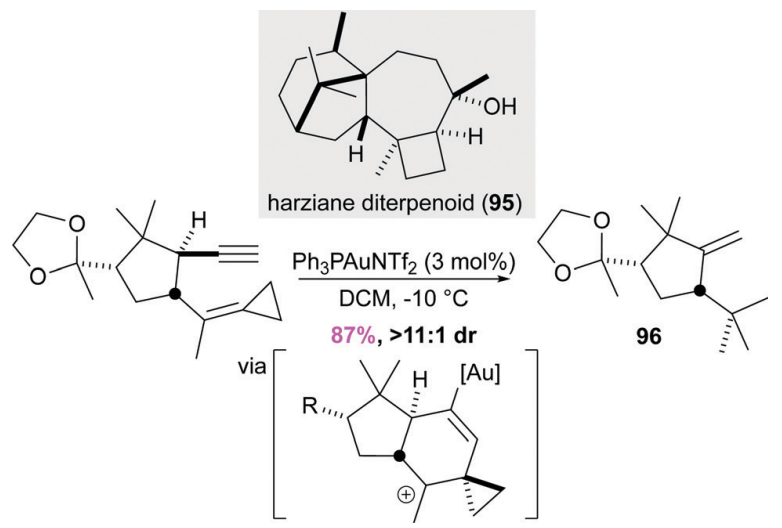

Scheme 30 Access to the tricyclic core of harziane diterpenoids (2020).

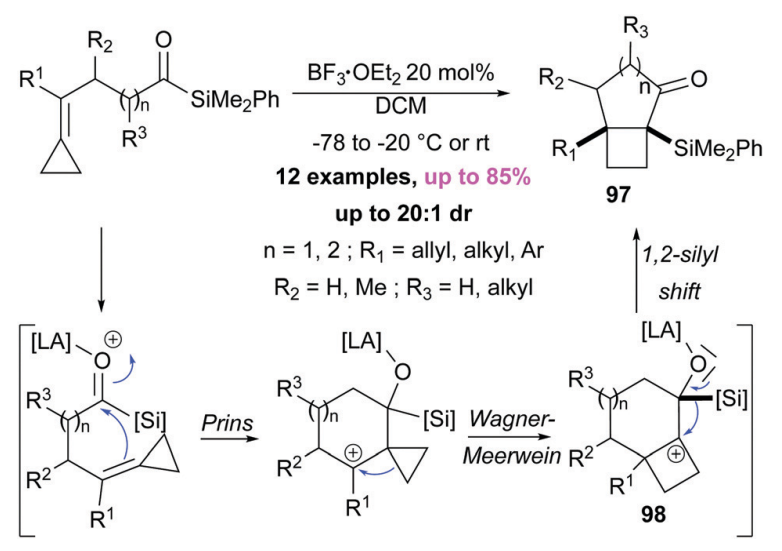

Scheme 31 Prins addition/ring expansion/1,2-silyl shift domino sequence (2020)

To conclude, the Wagner-Meerwein rearrangement of MCPs provides a stereocontrolled access to fused and spirocyclic polycyclic structures, in readiness for further applications.

3.2.2. Ring expansion of alkynyl cyclopropanes. A Rh(II)mediated chemoselective oxidative amination cascade of 1-(alkynyl)cyclopropyl methylsulfamates $\mathbf{9 9}$ have been reported by the group of $\mathrm{Shi}^{82}$ and allowed the construction of the corresponding four-membered rings fused to a heterocyclic system 100 (Scheme 32). Scope and limits of the reaction have been studied and the observed yields were between good to excellent. The electronic nature of the substituent on the alkynyl part did not seem to have any significant effect. Accordingly, the reaction tolerated both electron withdrawing and electron donating groups on the alkynyl part. Further calculation using Density-Functional Theory (DFT) by the same authors, gave insights into the reaction mechanism and they concluded, that the undertaken path involved a metallonitreneinitiated alkyne oxidation cascade. ${ }^{83}$

Both gold ${ }^{84}$ and ruthenium ${ }^{85}$ catalysts have also been utilised in the ring expansion of alkynylcyclopropanes 101 in presence of sulfonamides $\mathbf{1 0 2}$ to afford the corresponding 


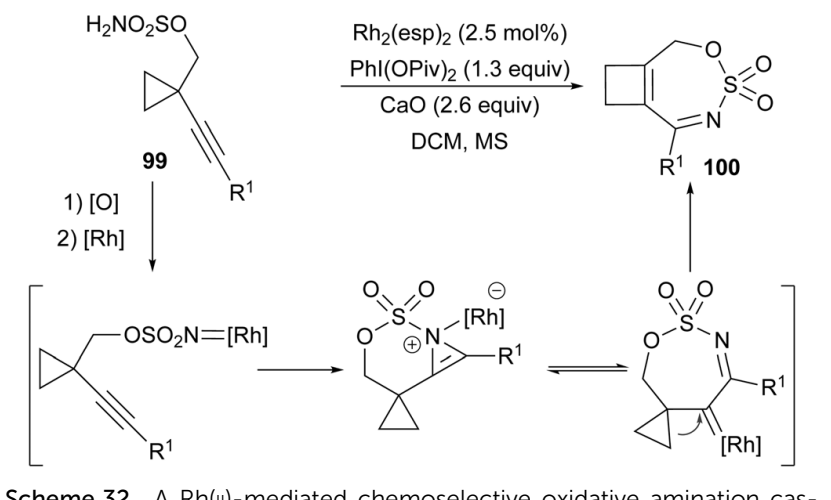

Scheme 32 A Rh(॥)-mediated chemoselective oxidative amination cascade (2017).

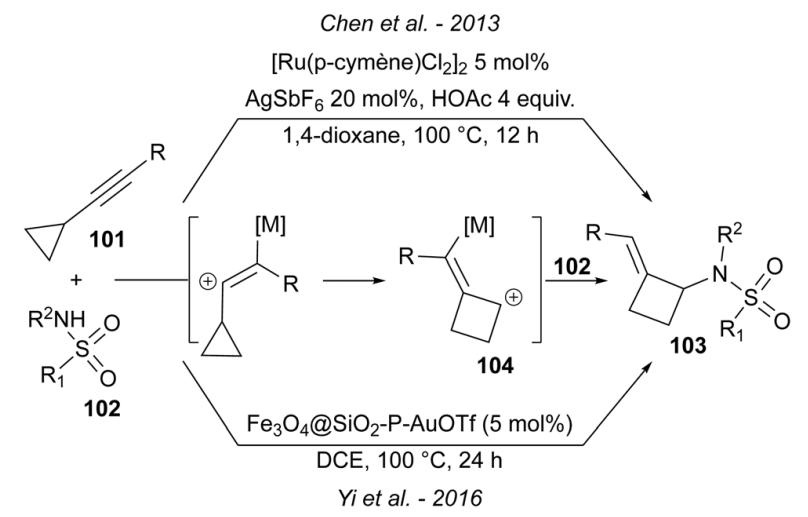

Scheme 33 Strategies towards $(E)$-2-alkylidenecyclobutanamines.

alkylidene-cyclobutanamides 103 (Scheme 33). After the ring expansion to the cyclobutane ring 104, the cationic species could be intermolecularly trapped by the sulfonamide $\mathbf{1 0 2}$. Interestingly, Yi et al. ${ }^{84}$ employed a heterogenous gold catalyst, therefore allowing its easy recycling and a procedure more environmentally friendly (Scheme 34).

3.2.3. [1,2]-Alkyl shift onto an intermediate carbenoid. Gold catalysis has also proven efficient in [1,2]-alkyl shift onto an intermediate carbenoid, kind of approach. For instance, Fensterbank described the cycloisomerisation of $O$-tethered 1,6-enynes 105 exhibiting, within the link, a four membered ring. ${ }^{86}$ The authors involved a ring expansion onto the transition-metal-stabilised carbocation intermediate 106. The corresponding fused tricyclic system containing a dihydropyran moiety 107 was obtained in good yields (except for unstable compound 107a $R_{1}=P h, R_{2}=H$, which decomposed only in few hours as a solution in deuterated chloroform). It was next demonstrated that a two-step sequence could deliver valuable macrocyclic ethers like 108, key structural units present in several natural products. ${ }^{87}$ Echavarren also extensively studied the gold-mediated Wagner-Meerwein rearrangement but in an intermolecular fashion. ${ }^{88}$ Satisfyingly, poly-substituted cyclobutenes $\mathbf{1 0 9}$ were obtained from very simple substrates, via the ring expansion of a gold carbene $\mathbf{1 1 0}$ (Scheme 35). It was thus
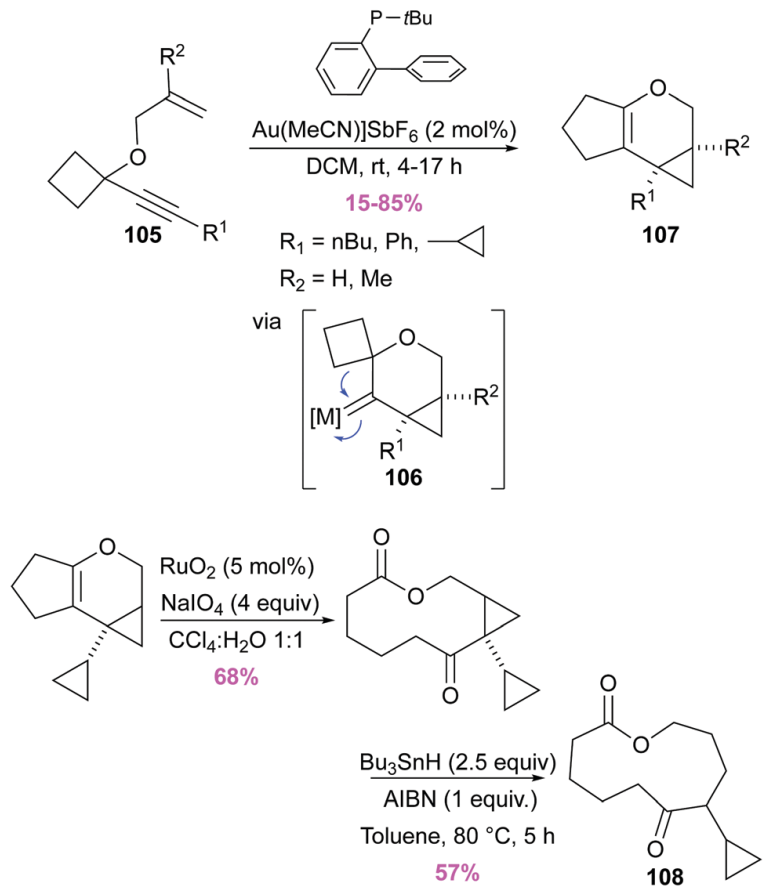

Scheme 34 Cycloisomerisation of O-tethered 1,6-enynes (2013).

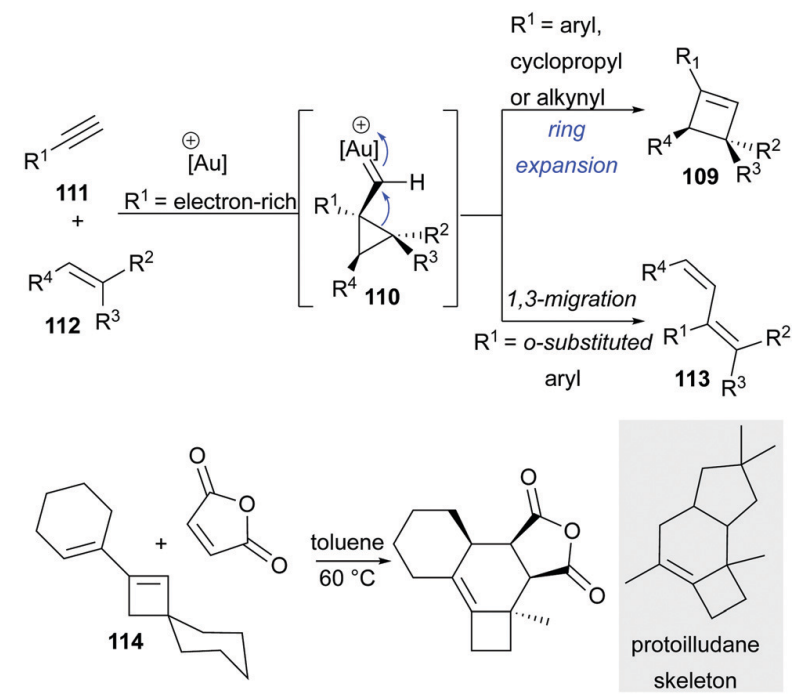

Scheme 35 Strategies towards poly-substituted cyclobutenes (2014).

demonstrated, that the formal [2+2] intermolecular cycloaddition reaction between electron-rich alkyne $\mathbf{1 1 1}$ and alkenes 112, occurred in very good yields. 1,3-Butadienes $\mathbf{1 1 3}$ have also been isolated in the case of ortho-substituted arylalkynes or electrondeficient alkynes. Mechanistically, a metathesis type path has been proposed. The course of this reaction has been the subject of extensive studies. ${ }^{88 b}$ Starting from 1,3-enynes, 1-vinyl-3substituted cyclobutenes ${ }^{89}$ could be obtained (see for instance 114). The latter served as key starting materials to more 


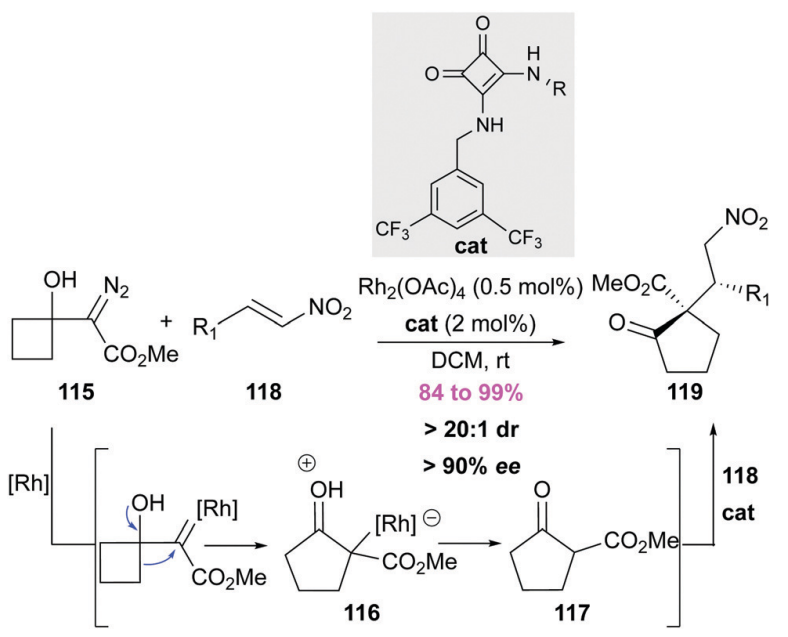

Scheme 36 Approach to $\alpha$-quaternary substituted cyclopentanones (2013)

complex structures like cyclopentenes, 2,3-dihydrofurans or even the protoilludane core.

A cyclobutanol exhibiting an $\alpha$-diazo derivative 115 was described in a paper published in 2013 by Chen et al. ${ }^{90}$ The diazo function was advantageously transformed into a rhodium-carbenoid intermediate, which could spontaneously undergo a rearrangement to the zwitterionic species 116 (Scheme 36). Thereafter, the urea-based squaramide catalyst could enable the enantioselective Michael addition of intermediate $\mathbf{1 1 7}$ onto a nitroolefin 118. This metal/chiral bifunctional organo combined catalysis allowed the formation of the corresponding cyclopentanone 119 in excellent yields (up to 99\%), with high diastereo- and enantioselectivities and, moreover, the creation of a quaternary stereocentre. The strategy employing a diazo function $\alpha$ to the cyclopropyl derivative, a well-known precursor of carbenoids, and as a mean to trigger the Wagner Meerwein rearrangement, had precedent in the literature. ${ }^{91}$ It was even utilised as a key step in the total synthesis of pipercyclobutanamide A and piperchabamide G. ${ }^{92}$ However, Gong's work is the first report of an enantioselective version. Additionally, the authors demonstrated the compatibility of the transition metal-based catalyst with a chiral bifunctional urea one.

To go even farther in this kind of approach, Tang reported a one-pot copper- and silver-catalysis allowing the ring expansion of alkynyl cyclopropanes $\mathbf{1 2 0}$ to highly substituted cyclobutenes 121 (Scheme 37). ${ }^{93}$ First, the copper catalyst could mediate the cycloaddition to the $N$-sulfonyl 1,2,3-triazole 122. This stable equivalent of diazo derivative could decompose to the corresponding carbenoid in presence of silver triflate. Finally, the Wagner-Meerwein rearrangement could be followed by an elimination step and an alumina oxide treatment would deliver the observed cyclobutenes 121.

An example reported last year by Aggarwal and co-workers, stood out from the crowd and could not be included in the above sub-sections. ${ }^{94}$ A Wagner-Meerwein ring expansion,

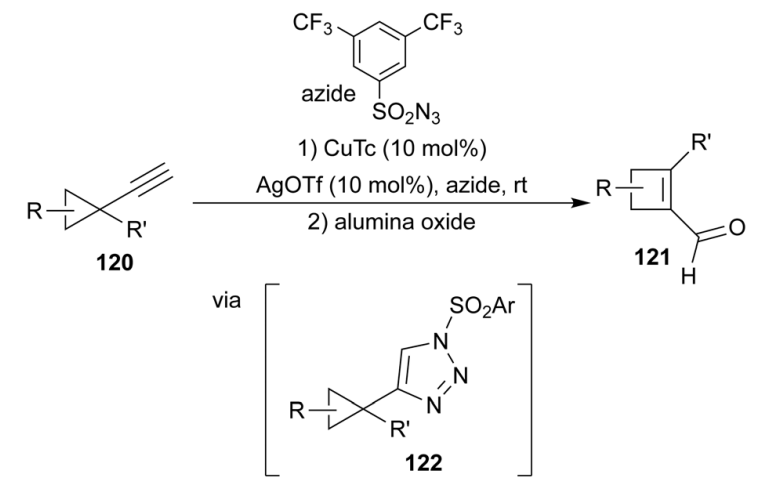

Scheme 37 One-pot ring expansion of alkynyl cyclopropanes (2013).

triggered by the electrophilic activation of vinylcyclopropyl boronate complexes $\mathbf{1 2 3}$ with an iminium salt, was elegantly accompanied by a 1,2-R-migration (Scheme 38 ). It was demonstrated that the reaction was compatible with both Grignard and organolithium reagents. The scope was broad and the 1,2substituted cyclobutyl boronic esters $\mathbf{1 2 4}$ were isolated in good yields, with high diastereoselectivites. From a structural point of view, spirocyclic scaffolds could be formed and cyclobutanes with up to three contiguous stereocentres were observed. Interestingly, the authors applied this approach to a short stereoselective synthesis of $( \pm)$-grandisol (125).

To summarise, the 1,2-alkyl migration for the ring enlargement of polyfunctionalised scaffolds is often part of a domino reaction. Such transformations have been elegantly applied to

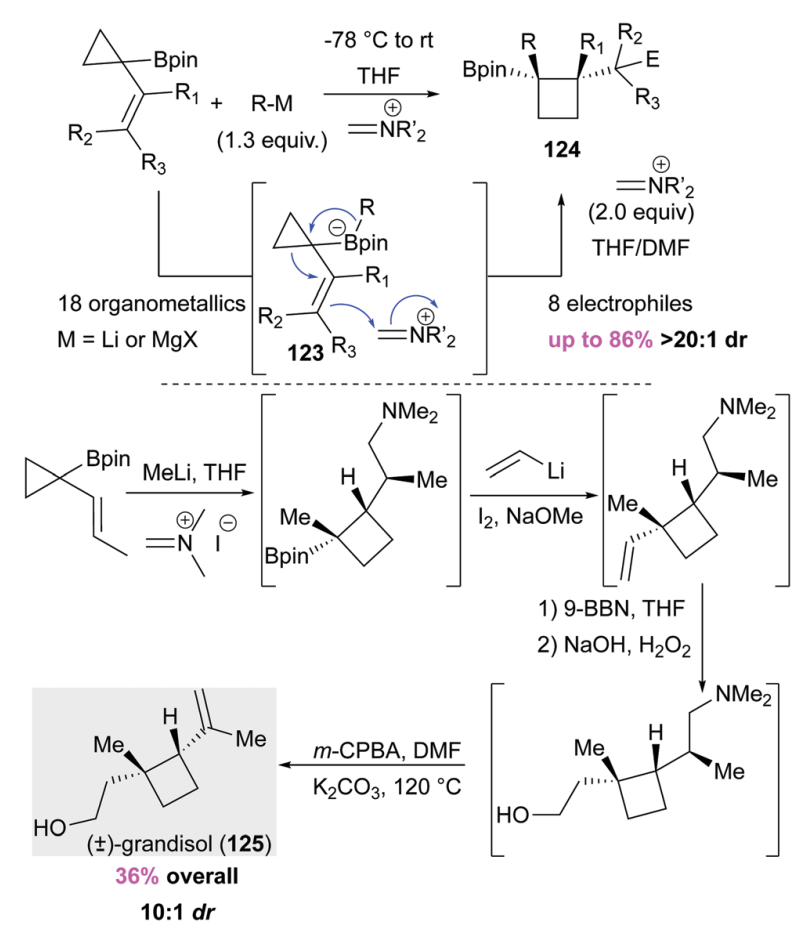

Scheme 38 Synthesis of cyclobutyl boronic esters (2020). 
the synthesis of synthetically challenging structures and can involve the formation, cleavage or migration of up to three $\mathrm{C}-\mathrm{C}$ or $\mathrm{C}-\mathrm{X}$ bonds. Efforts have also been carried on developing enantioselective processes. The vast majority of applications showcase the rapid build-up of complex structural motif such as quaternary carbon centres. It is also worth underlining that the field of 1,2-alkyl migration is largely dominated by gold catalysis. On one hand, the $\pi$-character of the $\mathrm{C}-\mathrm{C}$ bonds in a cyclopropane unit provides the kinetic opportunity to initiate the ring-opening, as do C-C multiple bonds through binding ${ }^{95}$ and, on the other hand, is responsible for its hyperconjugation. A stabilised non-classical carbocation is thus created.

\section{Group 3: pericyclic reactions}

The last part is devoted to the ring expansion of three- and fourmembered rings according to a pericyclic reaction pathway. A general scheme (Scheme 39) provides an overview of the different approaches at a glance. In other words, the ring expanded product generally results from either sigmatropic rearrangements ([1,3]-carbon shift, [3,3]-Cope rearrangement) or electrocyclic processes. In fine, the purpose of this section is to highlight the added value of using pericyclic reactions, to access challenging structural motif such as congested quaternary carbon centres, medium-size rings, spiro moieties.

\subsection{Sigmatropic rearrangements}

4.1.1. Cope rearrangement. With their $60^{\circ}$ bond angles (compared to the classical $109^{\circ}$ for typical $\mathrm{Csp}^{3}-\mathrm{Csp}^{3}$ bonds), cyclopropane units are small rings with considerable double bond character. Their reactivity can indeed be compared to that of alkenes. In that context, the divinylcylclopropane-cycloheptadiene rearrangement was first observed in 1960 by Vogel (Scheme 39). ${ }^{96}$ The thermal Cope rearrangement involving the ring opening of a strained cyclopropane became quite famous in the eighties. ${ }^{97}$ This catalyst-free versatile method for the formation of seven-membered rings possesses outstanding atom economy and, overall high degree of stereocontrol. Accordingly, the requirement of a boat transition state allows the impressive $\mathrm{C}-\mathrm{C} \sigma$-bonds formation in a highly stereospecific and predictable manner. ${ }^{98}$ Moreover, the transposition is driven by the release of ring strain and therefore occurs at significantly lower temperature than the classical acyclic 1,5dienes. This method has quickly found application in the synthesis of complex unnatural and natural products. However,
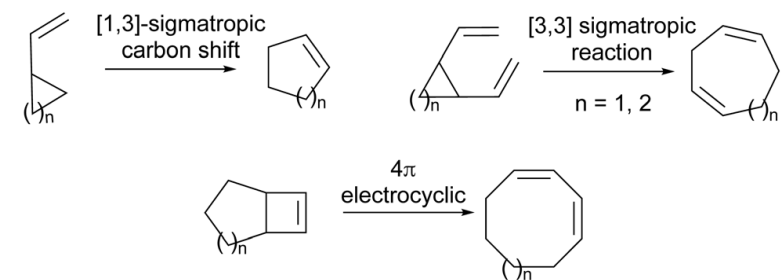

Scheme 39 Classical pericyclic reactions of cyclobutanes and cyclopropanes.

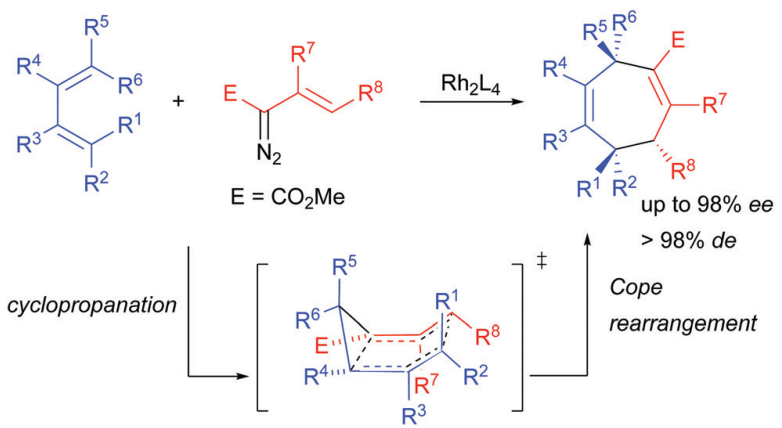

Scheme 40 Davies formal $(4+3)$ cycloaddition reaction.

one major drawback lied in the necessary construction of a cis relationship between the alkene substituents in the starting material, therefore lowering down the attractiveness of such a promising process. In 1998, Davies elegantly overturned this weakness by employing Donor-Acceptor diazo derivatives (Scheme 40). Not only a high diastereoselectivity and enantioselectivity in the Rh-mediated cyclopropanation step were secured, but also, by using a vinyl diazo derivative, the authors were able to perform the $[3,3]$-sigmatropic rearrangement in the same pot. ${ }^{99}$ This formal $(4+3)$ reaction set the stage for many elegant strategies and considerably shortened the path to molecular complexity. ${ }^{97 b, 100}$ More recently, Taylor studied the Cope rearrangement of gem-dimethyl substituted divinylcyclopropanes. ${ }^{101}$ The authors showed that the Cope rearrangement of trans-divinylcyclopropanes could take place given that higher reaction temperature, $130{ }^{\circ} \mathrm{C}$ in boiling xylenes for 17 hours, were utilized (Scheme 41, eqn (1)). From a mechanistic point of view, a trans-/cis-epimerisation of the divinylcyclopropane via a diradical cyclopropane opening, could first take place and, a subsequent Cope rearrangement could afford the corresponding 1,4-cycloheptadiene. The authors next demonstrated the inhibiting effects of the gemdimethyl substituents on the trans-/cis-isomerisation. The starting materials failed to deliver the product (Scheme 41, eqn (2)) but the corresponding cis-divinylcyclopropanes gave full conversion to the medium-size ring system (Scheme 41, eqn (3)).

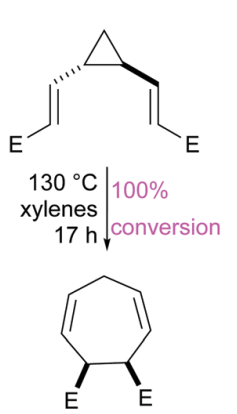

eq. 1

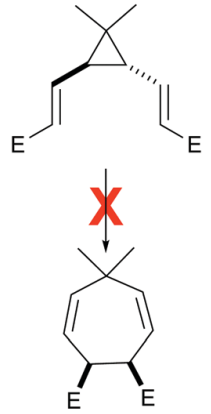

eq. 2

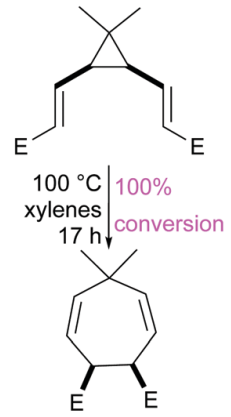

eq. 3
Scheme 41 Cope rearrangement of trans-divinylcyclopropanes (2013). 
OTBS

127

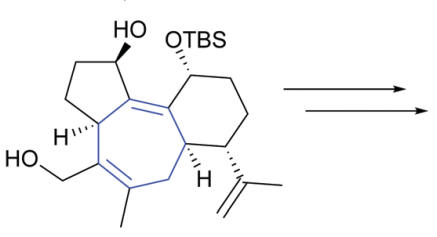

129

$31 \%$ overall yield

Scheme 42 Approach to the polycyclic core of curcusones (126) (2019).

In light of the impressive amount of work already described on the subject in the past decades, we decided to focus, in this section, on recent applications towards the total synthesis of natural products and/or creation of high molecular complexity via cascade reactions.

Stoltz reported very recently, an enantioselective approach toward the polycyclic core of curcusones (126) and used the divinylcyclopropane strategy as a key step to reach the sevenmembered ring (Scheme 42). ${ }^{102}$ Ring opening of lactone 127 in presence of DIBAL provided diol 128 together with trace amount of the desired ring expanded cycloadduct 129. Accordingly, gentle heating $\left(50{ }^{\circ} \mathrm{C}\right)$ of the crude mixture in benzene pleasingly converted to completion the remaining cisdivinylcyclopropane, thanks to a Cope rearrangement. The tricyclic system was obtained in $31 \%$ overall yield. A boat transition state set the relative stereochemistry at the junction of the seven- and five- and six-membered rings respectively. The expected compound was delivered as a unique diastereomer. Interestingly, this rearrangement required a prior strain release to be effective. In other words, the presence of the lactone tethering the system, allowed to temporarily mask the divinylcyclopropane function.

The same group has also been interested, for few years now, in the furanobutenolide-derived polycyclic norcembranoid diterpenes. ${ }^{103}$ All approaches rely on a diastereoselective Cope rearrangement. More precisely, they developed a straightforward access to the $[6,7,5,5]$ tetracyclic core of ineleganolide (130). ${ }^{104}$ Three complementary domino sequences were reported by the authors. To get straight to the point, the divinylcyclopropane $\mathbf{1 3 1}$ could not undergo, as anticipated, a direct Cope rearrangement since it would lead to a highly strained embedded cycloheptadiene 132, presenting two anti Bredt bridgehead alkene (Scheme 43). However, if 131 was treated with lithium hydroxide in cold and wet THF, first an acetate cleavage could take place, followed by a translactonisation disrupting the bridged system. Finally, the [3,3]-sigmatropic rearrangement of the less strained three-membered ring 133 could provide the desired intricated tetracyclic structure $\mathbf{1 3 4}$ in $55 \%$ yield. During this domino sequence featuring a small-ring
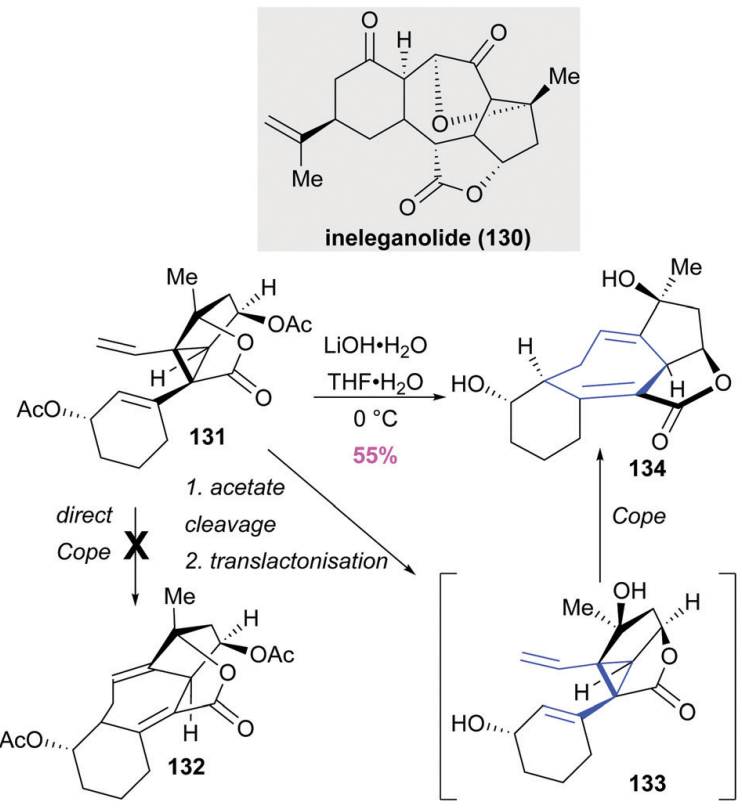

Scheme 43 Access to the $[6,7,5,5]$ tetracyclic core of ineleganolide $(\mathbf{1 3 0})$ (2017).

expansion, two single bonds and a tertiary stereogenic centre were created with total diastereoselectivity. Importantly, a transcycloheptadiene was reached, representing the first reported synthesis of the $(6,7,5,5)$ tetracyclic core of ineleganolide $(\mathbf{1 3 0})$. This transformation was "effortless" since such a framework simply and spontaneously rearranged.

In 2012, a concise route to the core of molecules isolated from the Schisandra genus was reported [see for instance lancifodilactone $\mathrm{F}$ (135) \& micrandilactone $\mathrm{B}$ (136)]. ${ }^{105} \mathrm{~A}$ domino cascade reaction featuring a Pd-mediated Sonogashira coupling/lactonization/Cope rearrangement cascade onto diastereomer 137 allowed the formation of the ABC skeleton 138 of lancifodilactone $\mathrm{F}$ (135) in $31 \%$ yield, as a single diastereomer (Scheme 44). The sequence during which three single bonds and two stereogenic centres, including a tetracyclic carbon, were created, was thus validated. The diastereoselectivity of the reaction came from: the controlled formation of the $Z$-exocyclic double bond during the 5-exo-dig lactonisation (see 139), followed by a boat transition state for the Cope rearrangement (see 140). A further one-pot deprotection-Michael addition gratifyingly delivered the desired tetracyclic backbone 141 of micrandilactone B (136) in 64\% yield.

The gold- or platinium-catalysed cycloisomerisation of dienyne as part of a cascade sequence with the subsequent divinylcylclopropane-cycloheptadiene rearrangement, has been described on several occasions and all papers related to the subject, elegantly set polycyclic structures exhibiting a cycloheptadiene. ${ }^{106}$ Newcomb et al., in their effort to synthesize the $( \pm$ )-gelsenicine (142) natural product, for the first time, also took advantage of a bicyclo[4.1.0]heptene 143, arising from a gold-catalysed cycloisomerisation key step (Scheme 45). ${ }^{107}$ 

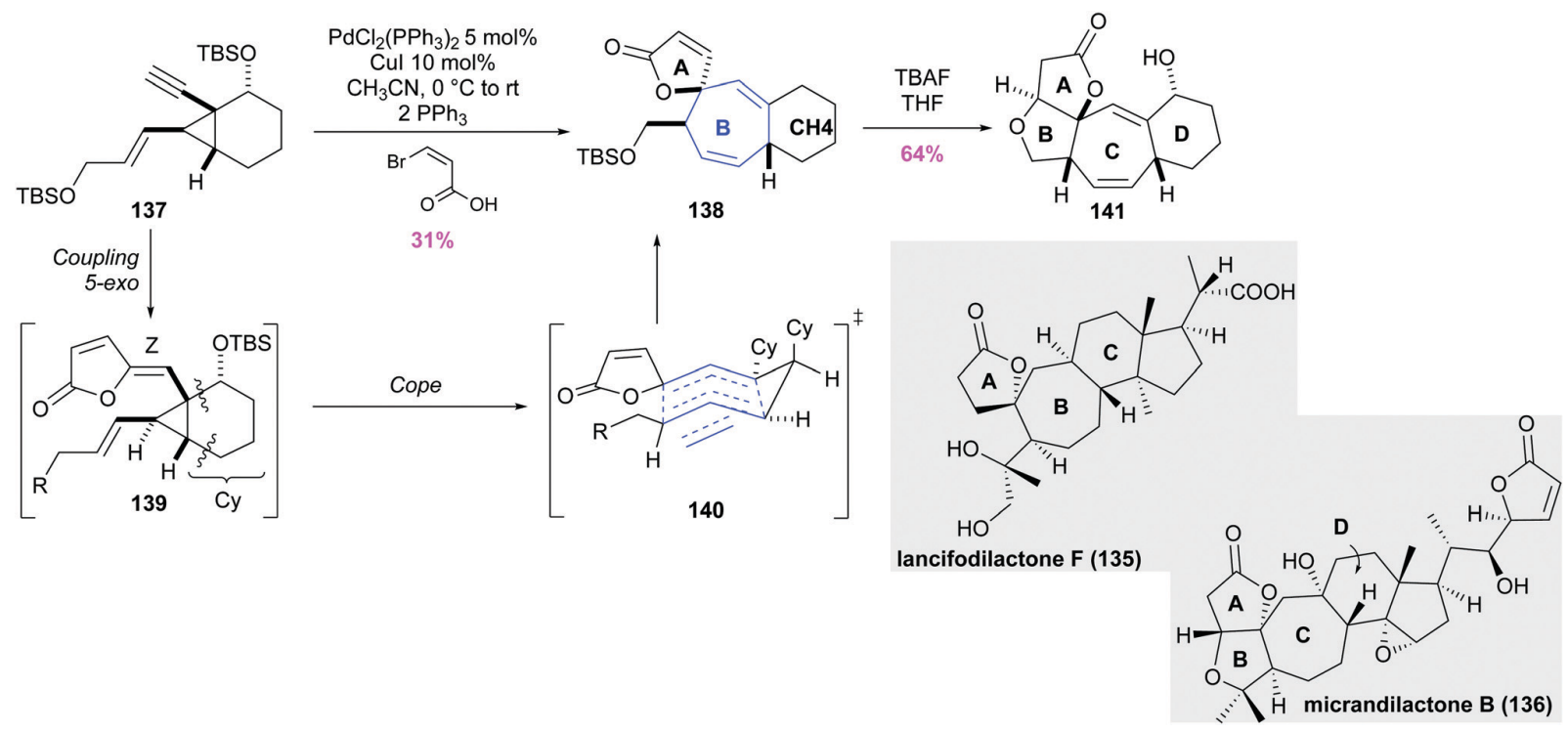

Scheme 44 A concise route to the core of molecules isolated from the Schisandra genus (2012).

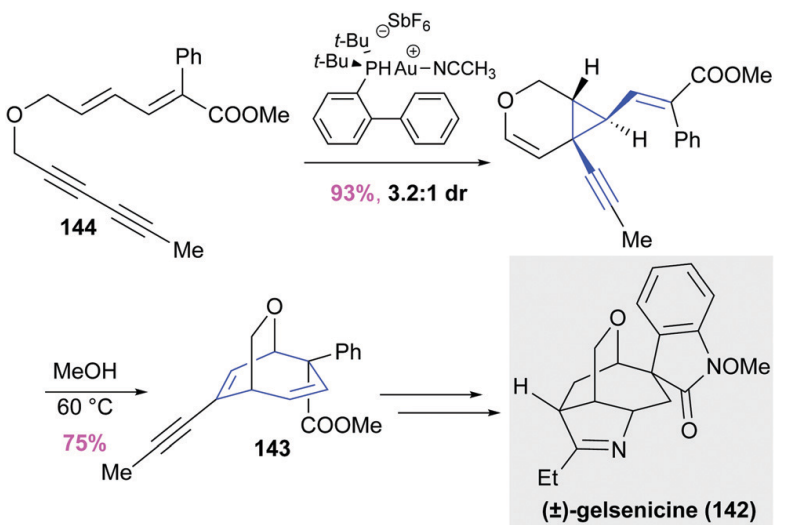

Scheme 45 Approach towards ( \pm )-gelsenicine (142) (2015).

The target was reached in 13 steps on the longest linear sequence and, remarkably, it is a completely protectinggroup-free approach. The authors chose to run a stepwise strategy on $(E, E)$-dienyne $\mathbf{1 4 4}$ to reach higher diastereoselectivity $(3.2: 1 \mathrm{dr})$. However, it should be underlined that a onepot reaction cascade was also feasible and led as well to the desired polycyclic structure $143(83 \%, 1.9: 1 \mathrm{dr})$.

Another approach starting also from dienyne, was reported by Chen. ${ }^{108}$ The authors set an elegant formal gold mediated $(4+2+1)$ cycloaddition reaction to access [5.3.0] substituted bicyclic compounds. The intermolecular reaction between dienyne 145 and a donor-acceptor diazo compound 146 in presence of a NHC-Au(I) catalyst, provided adduct 147 in very good yields (Scheme 46). More importantly, this cascade reaction allowed the challenging diastereoselective formation of a quaternary centre. Nevertheless, the scope of the reaction is limited to aryl groups on the diazo derivative. However,

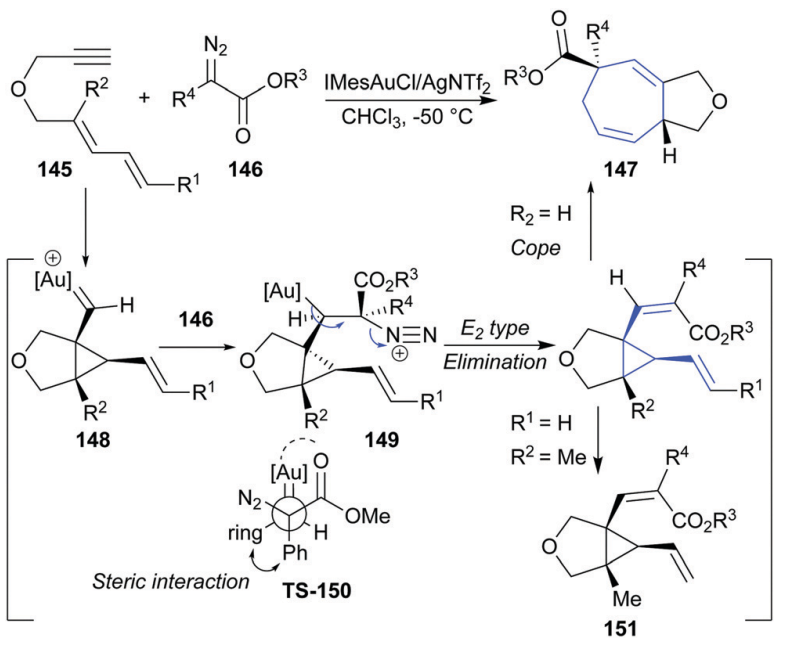

Scheme 46 A formal intermolecular [4+2+1] cycloaddition (2020)

compounds bearing electron-withdrawing or electrondonating groups on the phenyl substituent showed similar reactivity, indicating that the electronic nature does not play an essential role in the transformation. Hypothetically, the nucleophilic addition of the diazo derivative 146 on the gold intermediate 148 should lead to intermediate 149 via TS-150. According to the authors, the latter is the most favoured one because of reduced steric interaction and possible $\mathrm{Au}-\mathrm{O}$ interaction. We believe the presence of a donor component (aryl group) on the diazo derivative to be essential to increase the nucleophilicity of the diazo compound, thus, explaining why the simplest ethyldiazoacetate did not react. Finally, an elimination step followed by the Cope rearrangement could deliver the bicyclic structure 147. The reaction is quite sensitive to 

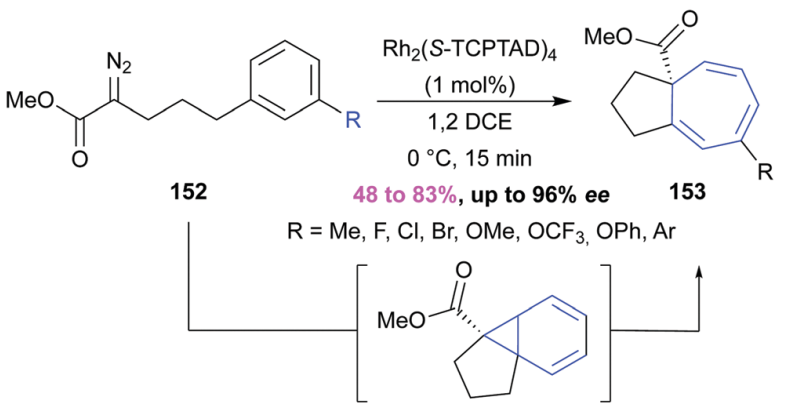

Scheme 47 A Buchner ring expansion example (2019).

steric encumbrance, since substitution on the diene required harsher reaction conditions ( 24 hours in boiling toluene), for the $[3,3]$-sigmatropic rearrangement to occur. Otherwise, the divinylcyclopropane $\mathbf{1 5 1}$ is instead isolated.

The reaction of diazocarbonyl derivatives with arenes (otherwise known as Buchner ring expansion) can be thought of as a special case of divinylcyclopropane rearrangement. This onepot, two-step reaction has been thoroughly described ${ }^{109}$ and was recently reviewed. ${ }^{110}$ It has been part of elegant reported total syntheses. ${ }^{111}$ A current example was devoted to the asymmetric synthesis of $(5,7)$-fused ring systems. ${ }^{112}$ The authors focused their efforts on finding conditions: (i) preventing the formation of the so-called $\beta$-elimination side products and (ii) providing the highest possible enantiomeric excesses. The intramolecular rhodium-mediated cyclopropanation of the arene part $\mathbf{1 5 2}$ followed by a ring-opening to the cycloheptatriene $\mathbf{1 5 3}$ set the challenging stereochemistry of the quaternary carbon centre at the ring junction in good yields and competitive enantioselectivity (Scheme 47).

Preparation of cyclooctadiene-containing systems through Cope rearrangement from divinylcyclobutanes this time, is also a well-known process ${ }^{113}$ and is often obtained via a [2+2] cycloaddition of 1,3-dienes. ${ }^{114}$ This kind of approach has proven useful in the total synthesis of natural products. ${ }^{115}$ Herein, we wish to highlight the elegant and original work reported from the group of Daugulis. ${ }^{116}$ En route to helicene, they developed a strategy starting from phenols. When the
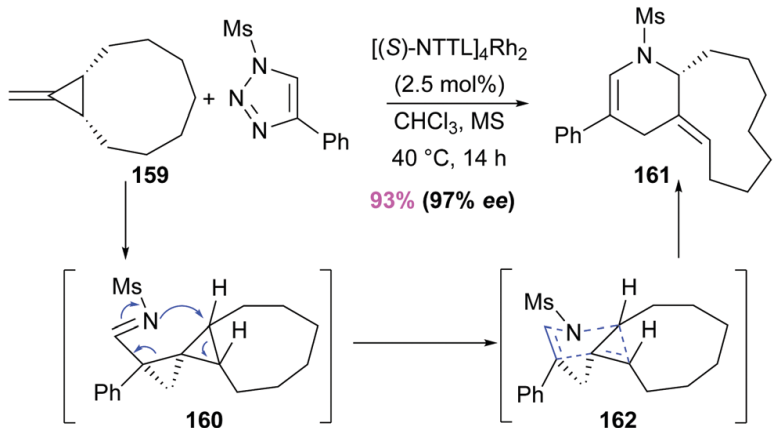

Scheme 49 A retro-Claisen type rearrangement to 10-trans-cycloalkene (2014).

latter was submitted to four equivalents of chlorobenzene in presence of LiTMP in a THF/pentane mixture at room temperature, the corresponding helicene $\mathbf{1 5 4}$ was obtained in $67 \%$ yield (Scheme 48). The mechanism suggested by the authors could involve a $[2+2]$ reaction between benzyne and phenolate affording the four-membered ring 155. Next a Cope rearrangement of the divinylcyclobutane 155 could furnish the eight-membered ring 156 which, after reacting with a second equivalent of benzyne could deliver the tetracyclic structure 157. Ring opening of the latter could deliver the 10-membered ring ketone 158. Finally, a ring contraction/dehydration sequence could afford the observed helicene 154. A similar electrocyclic ring opening leading to benzocyclooctatrienones has also been reported by Yamamoto, and featured a one-pot ruthenium-catalysed cyclisation-iodine-mediated oxidative ring expansion. ${ }^{117}$

4.1.2. Other sigmatropic rearrangements. In line, with the Cope rearrangement that we just mentioned, we wanted to first turn our attention to another [3,3] Sigmatropic rearrangement, namely the retro-Claisen type rearrangement. Miura and Murakami proposed a sequence to trans-cycloalkenes via an enantioselective cyclopropanation and a ring expansion. ${ }^{118}$ This impressive transformation set the stage for the asymmetric formation of 8- and 10-membered trans-cycloalkenes fused with dehydropiperidine (Scheme 49). This one-pot, two step procedure, featured first an enantioselective cyclopropanation onto the MCP derivative 159, thanks to a chiral rhodium catalyst. The resulting

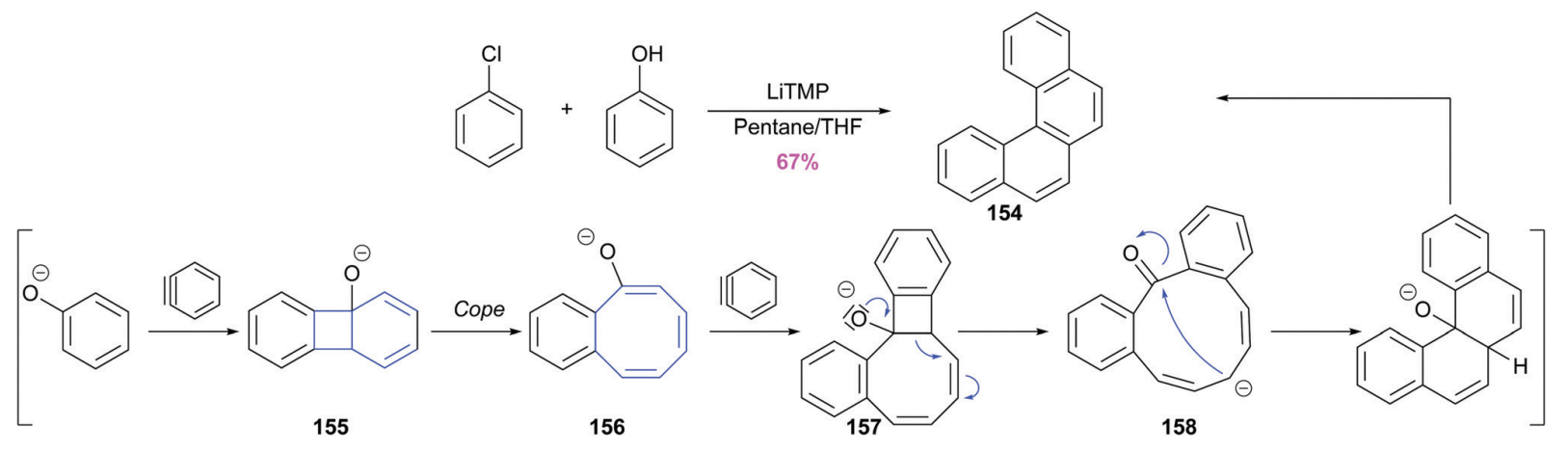

Scheme 48 Synthesis of helicenes (2013). 


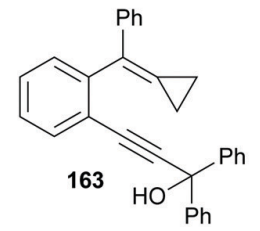<smiles></smiles>

$\mathrm{Nu}$ : substituted indole
TFA (20 mol\%)

$$
\mathrm{PhCl}, \mathrm{rt}
$$

9 examples, $40-69 \%$

Ratios 164:167 1:1 to $2.4: 1$

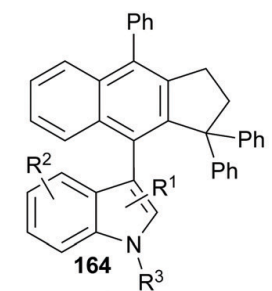

formal $\uparrow 1,3]$-alkyl

shift

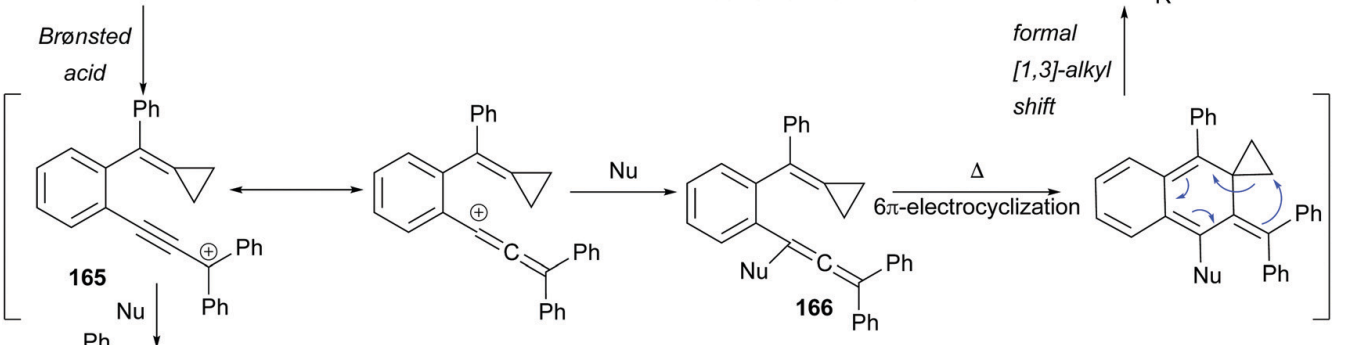

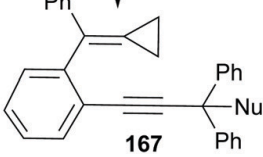

Scheme 50 Approach to cyclopenta[b]naphthalenes (2020).

spiro[2.2]pentane 160 could then undergo a concerted skeletal rearrangement to $\mathbf{1 6 1}$. The resulting trans-alkene would originate from a closed chair-like transition state $\mathbf{1 6 2}$. The reported yields were excellent and high enantiomeric excesses were observed.

The interesting biological activity profile and structural complexity of cyclopenta[ $[b]$ naphthalene moieties have drawn significant attention ${ }^{119}$ and Shi proposed a straightforward access to this kind of structural motifs starting from the famously strained and reactive methylenecyclopropenes. ${ }^{120}$

Under acidic conditions and in presence of a nucleophile (like indole or pyrrole) in the reaction mixture, the propargyl alcohol 163 underwent a cascade reaction to the polyaromatic structure 164 (Scheme 50). First, the carbocationic intermediates 165 could react with pyrrole (or indole) at the 2- or 1-position leading to intermediate 166 or side product 167 . Upon heating a $6 \pi$ electrocyclisation followed by a formal $[1,3]$ sigmatropic alkyl shift driven by a rearomatisation could provide the desired cyclopenta[$[b]$ naphthalene skeletons $\mathbf{1 6 4}$ in very good yields.

The recent strategic applications of the sigmatropic rearrangements have been described. They all showcase the significant role of this type of ring expansion in the design of efficient and stereocontrolled synthetic routes. The most appealing approaches started from acyclic starting materials. The corresponding smallsize ring were obtained as intermediates and "spontaneously" rearranged into more elaborated polycyclic scaffolds. Overall this process is less time-consuming and combined pot, atom and step economy (PASE). ${ }^{121}$ It is definitely a source of inspiration for further utilisation, notably in natural product synthesis, or the design and development of novel materials.

\subsection{Other pericyclic reactions}

Assembling complex molecular scaffolds as quickly and as efficiently as possible, is the holy grail of each synthetic organic chemist. Pericyclic reactions and electrocyclic reactions more precisely, is a mean to meet these criteria.

Substituted benzoic acids are quite appealing starting materials for the synthesis of many complicated naturally occurring compounds. $^{122}$ In 2015, Magauer developed an interesting strategy to access the 3-hydroxybenzoate subunit. ${ }^{123}$ Previously reported methods suffered from poor selectivity and/or low reactivity. ${ }^{124}$

For those reasons, the authors came up with an unprecedented approach relying on a thermal $2 \pi$ electrocyclic disrotatory ring opening of bicyclo[3.1.0]hexane-2-ones followed by an aromatisation step. The bicyclic starting material 168 is easily available from the cyclopropanation of the corresponding cyclopentenone in presence of cheap reagents, namely the lithium enolate of methyl dichloroacetate. The authors quickly demonstrated that the (cis,cis) isomer of $\mathbf{1 6 8}$ was reluctant to undergo the thermally allowed cyclopropyl allyl cation type $2 \pi$ disrotatory ring opening. Whereas, the (cis,trans)-168 cyclopropane, when heated up to $190{ }^{\circ} \mathrm{C}$ in the polar solvent sulfolane, quickly delivered the corresponding 3-hydroxybenzo-ate 169 in excellent yields and regioselectivity (Scheme 51). This transformation turned out to be compatible with a wide range of substituents and could be extended to the synthesis of anilines 170 or phenols 171, when amines or alcohols were respectively used as condensation reagents of the carbonyle group. Later on, the authors showcased their methodology in the total synthesis of the natural product salimabromide $(\mathbf{1 7 2})^{125}$ and the polycyclic core of chartarin (173). ${ }^{126}$

Polysusbstituted phenols are ideally poised for diverse synthetic elaborations by manipulation of their functional groups (for instance, oxidation to the ubiquitous quinones) or by oxidative phenolic couplings. Recently, $\mathrm{Hu}$ and coworkers applied a step-economic approach to hydroquinones, reported 

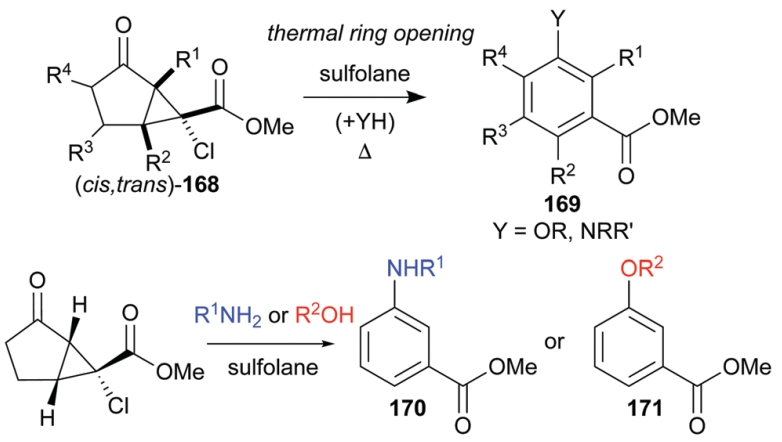

$\mathrm{R}^{1}=\mathrm{Bn}(70 \%) ; \mathrm{Ph}(83 \%) ;$ piperidine (81\%); (allyl) 2 (73\%)

$\mathrm{R}^{2}=\mathrm{Bn}(78 \%) ; \mathrm{Ph}(42 \%)$; cyclopentanyl (96\%); pentyl (89\%)
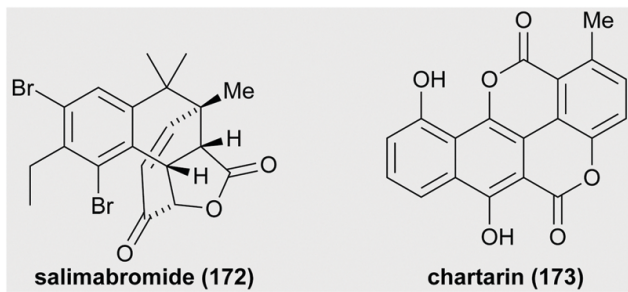

Scheme 51 A $2 \pi$-electrocyclic disrotatory ring opening (2015).

by Moore ${ }^{127}$ and Liebeskind in the late eighties. ${ }^{128}$ The authors used this $4 \pi$-electrocyclic ring-opening $/ 6 \pi$ electrocyclisation cascade for the total synthesis of three naturally occurring products: $( \pm)$-diperezone (174), parvistemin A $(\mathbf{1 7 5})^{129}$ and $\left( \pm\right.$ )-cereoanhydride (176). ${ }^{130}$ The thermally favoured $4 \pi$ electrocyclic conrotatory ring opening of cyclobutenone 177 could lead to intermediate $\mathbf{1 7 8}$ which, upon $6 \pi$ ring-closure and tautomerisation could deliver the corresponding hydroquinones 179 (Scheme 52). A subsequent DDQ oxidation thereafter furnished quinones 180 in $40 \%$ yield thus demonstrating the synthetic potential of substituted cyclobutenediones. ${ }^{131}$ The natural product $( \pm)$-cereoanhydride (176) was obtained in nine steps only, therefore demonstrating how electrocyclic cascade reactions could shorten the path to complicated molecular structure.

In 2015, Arichi et al. focused their attention on a ring expansion cascade for the synthesis of medium-size ring system starting from a fused cyclobutene (Scheme 53) ${ }^{132}$ When cyclobutenes 181 were treated with an iodoaniline derivative in presence of a palladium catalyst in toluene at $100{ }^{\circ} \mathrm{C}$, the cyclooctene- and even the cyclononene-fused indolines 182 were isolated in yields ranging from 45 to $80 \%$. The thermal stereospecific conrotatory $4 \pi$ electrocyclic ring opening of the cis-fused cyclobutenes would lead to the corresponding cis,trans-cycloalkadienes 183. Then, a $\mathrm{Pd}(\mathrm{II})$ complex generated from the oxidative addition of a $\operatorname{Pd}(0)$ into the carbonhalogen bond of the iodoaniline reactant, would be involved in a Heck arylation. Interestingly, the authors demonstrated a point-to planar-to point chirality transfer via the transient planar chiral intermediate 183. Another torquoselective $4 \pi$ electrocyclic conrotatory ring opening was utilised this time on cyclobutenamides as part of a cascade reaction, and

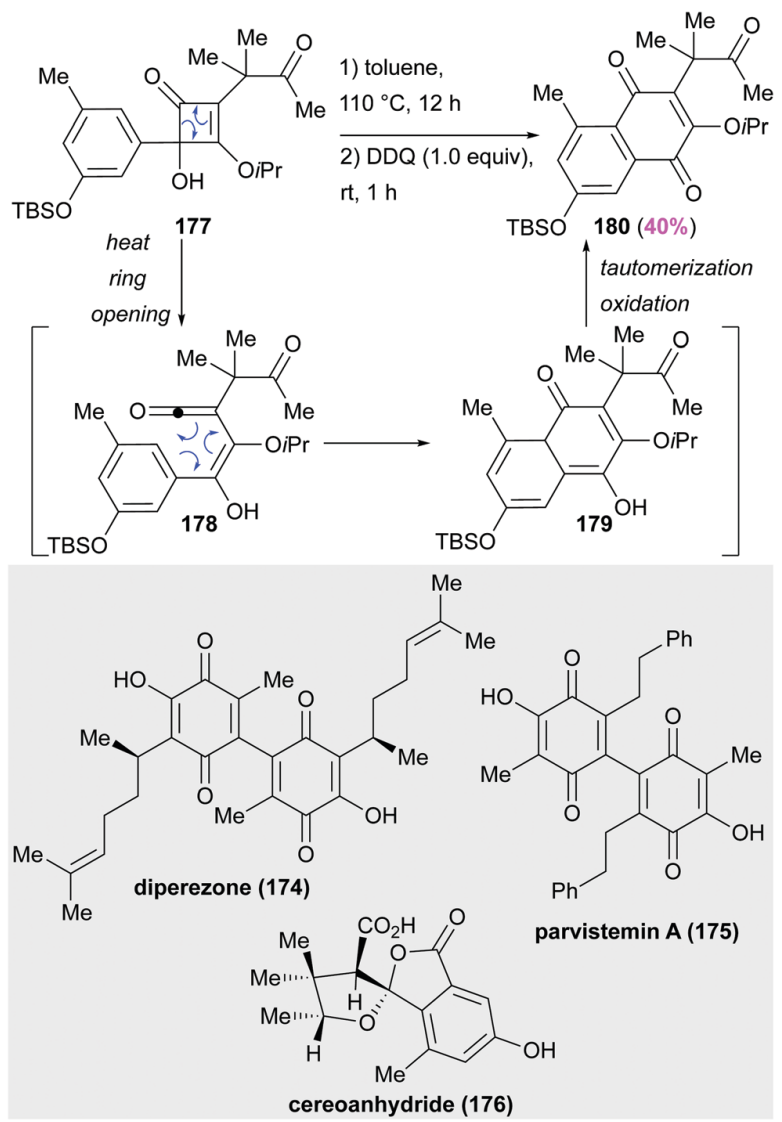

Scheme 52 A $4 \pi$-electrocyclic ring-opening $/ 6 \pi$-electrocyclisation cascade (2016).

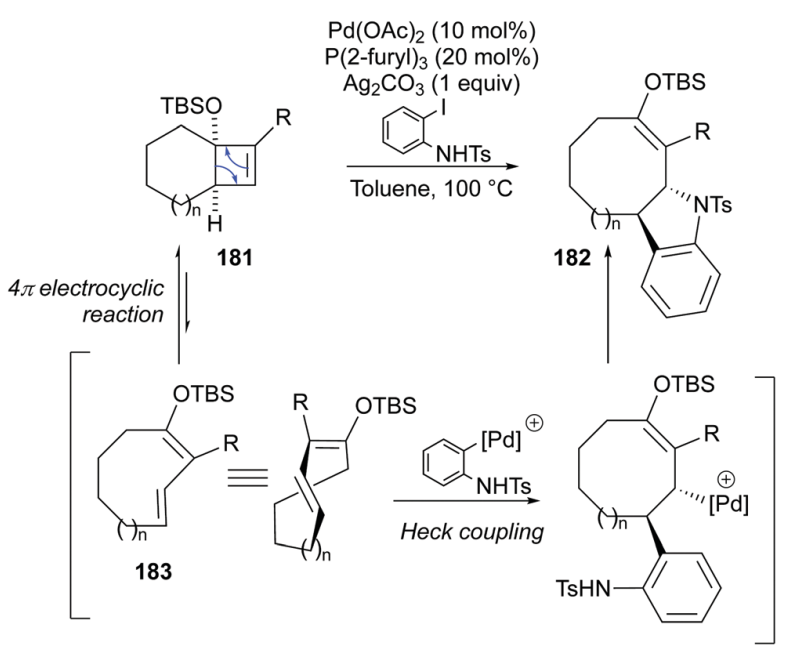

Scheme 53 Thermal stereospecific conrotatory $4 \pi$-electrocyclic ring opening (2015).

interestingly involved a strained cis,trans-cycloheptadienone intermediate. ${ }^{133}$ The $\mathrm{AlCl}_{3}$-Lewis acid mediated the ring expansion of 4,5-fused cyclobutenamides $\mathbf{1 8 4}$ to the strained 

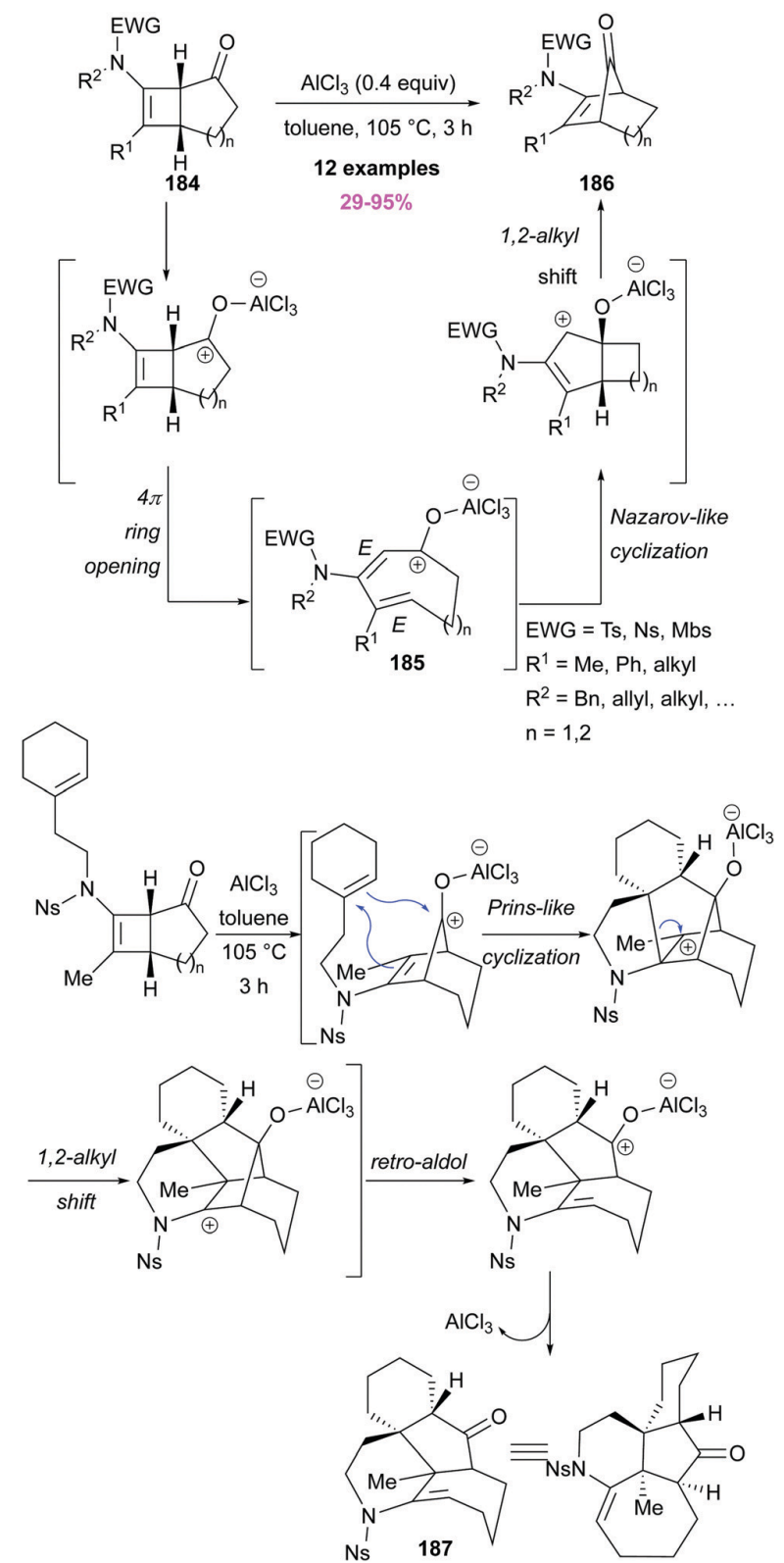

Scheme 54 A $4 \pi$-electrocyclic conrotatory ring opening to medium-size bridged systems (2015).

medium-size ring 185 (Scheme 54). Thereafter a Nazarov-like cyclisation followed by a 1,2-alkyl shift provided the [2.2.1] bicyclic ketones 186 in excellent yields. This reaction could also be applied to the 4,6-fused cyclobutenamides $(\mathbf{1 8 4}, n=2)$ and therefore furnished the corresponding eight-membered ring adduct. Finally, the authors demonstrated the synthetic potential of their strategy thanks to the synthesis of polycyclic frameworks in one step only and starting from readily available cyclobutenamides (see the preparation of $\mathbf{1 8 7}$, for instance).

The cyclic nature of the transition state of pericylic reactions, associated with the inherent ring strain of cyclopropanes or cyclobutanes, is a clear asset in terms of stereo- and regiocontrol. It is also an efficient trick to reach quaternary centres, which are common structural motifs in many bioactive natural products and pharmaceuticals. The construction of all-carbon quaternary centres has indeed received special attention from the synthetic community. ${ }^{134}$

\section{Conclusions}

This review intended to highlight the tremendous power and versatility of small ring systems. Cyclobutanes and cyclopropanes derivatives have drawn increasing attention because they constitute valuable building blocks. Their rearrangement reactions represent one of the most attractive applications as they allow enantioselective and diastereoselective formations of chemical bonds, especially in the creation of sterically congested quaternary or tetrasubstituted carbon centre. The presence of multiple functionalities in the products allows further transformations, therefore giving access to more complex structures. For the above-mentioned reasons, recent applications have been found in the total synthesis of natural products. In addition, the ring expansion as a part of a domino cascade reaction seems to be the general trend observed lately, and allow an elegant access to intricate molecular architectures.

To conclude, this small ring expansion strategies clearly have a bright future in synthetic chemistry, since they give birth to libraries of more complex structures that would be impossible or difficult to attain by more classical approaches.

\section{Conflicts of interest}

There are no conflicts to declare.

\section{Acknowledgements}

Financial support from Aix Marseille University, Centrale Marseille, the CNRS and, the Agence Nationale pour la Recherche (ANR 18-CE07-0005) are gratefully acknowledged.

\section{Notes and references}

1 (a) P. A. Wender, Nat. Prod. Rep., 2014, 31, 433-440; (b) O. O. Grygorenko, D. M. Volochnyuk, S. V. Ryabukhi and D. B. Judd, Chem. - Eur. J., 2020, 26, 1196-1237; (c) C. A. Kuttruff, M. D. Eastgate and P. S. Baran, Nat. Prod. Rep., 2014, 31, 419-432; (d) J. Mulzer, Nat. Prod. Rep., 2014, 31, 595-603.

2 (a) R. D. Bach and O. Dmitrenko, J. Am. Chem. Soc., 2004, 126, 4444-4452; (b) R. D. Bach and O. Dmitrenko, J. Org. Chem., 2002, 67, 2588-2599; (c) T. Dudev and C. Lim, J. Am. Chem. Soc., 1998, 120, 4450-4458.

3 A. de Meijere, J. Am. Chem. Soc., 1979, 18, 819-826.

4 For reviews on cyclopropane and cyclobutane strategies in natural product synthesis, see for instance: (a) C. A. Carson 
and M. A. Kerr, Chem. Soc. Rev., 2009, 38, 3051-3060; (b) J. C. Namyslo and D. Kaufmann, Chem. Rev., 2003, 103, 1485-1538; (c) F. D. Simone and J. Waser, Synthesis, 2009, 3353-3374; (d) M. Honma, H. Takeda, M. Takano and M. Nakada, Synlett, 2009, 1695-1712; (e) F. D. Simone and J. Waser, Synlett, 2011, 589-593; $(f)$ D. Zhang, H. Song and Y. Qin, Acc. Chem. Res., 2011, 44, 447-457; $(g)$ P. Tang and Y. Qin, Synthesis, 2012, 2969-2984; (h) N.-Y. Fu, S.-H. Chan, S.-H. Wong and N. C. Henry, in The chemistry of functional groups, ed. Z. Rappoport and J. F. Liebman, Patai Series, 2005, pp. 357-440.

5 W. R. J. D. Galloway, A. Isidro-Llobet and D. Spring, Nat. Commun., 2010, 1, 1-13.

6 (a) J. R. Donald and W. P. Unsworth, Chem. - Eur. J., 2017, 23, 8780-8799; (b) K. Prantz and J. Mulzer, Chem. Rev., 2010, 110, 3741-3766; (c) T. C. Stephens and W. P. Unsworth, Synlett, 2020, 133-146; (d) A. K. Clarke and W. P. Unsworth, Chem. Sci., 2020, 11, 2876-2881.

7 D. J. Mack and J. T. Njardarson, ACS Catal., 2013, 3, 272-286.

8 T. Seiser, T. Saget, D. N. Tran and N. Cramer, Angew. Chem., Int. Ed., 2011, 50, 7740-7752.

9 X. Cai, W. Liang and M. Dai, Tetrahedron, 2019, 75, 193-208.

10 (a) P. Natho, L. A. T. Alle and P. J. Parsons, Tetrahedron Lett., 2020, 61, DOI: 10.1016/j.tetlet.2020.151695.; (b) J. Le Bras and J. Muzart, Tetrahedron, 2020, 76, DOI: 10.1016/ j.tet.2019.130879.

11 M. Hesse, in Ring enlargement in Organic Synthesis, ed. M. G. Weller, Weinheim, New-York, Basel, Cambridge: VCH, 1991.

12 R. A. Panish, S. R. Chintala and J. M. Fox, Angew. Chem., Int. Ed., 2016, 55, 4983-4987.

13 The enantioselective bicyclobutanation was independently reported by Davies and Fox: (a) C. Qin and H. M. L. Davies, Org. Lett., 2013, 15, 310-313; (b) R. Panish, S. R. Chintala, D. T. Boruta, Y. Fang, M. T. Taylor and J. M. Fox, J. Am. Chem. Soc., 2013, 135, 9283-9286.

14 A. Fawcett, Pure Appl. Chem., 2020, 92, 751-765.

15 H.-D. Sun, S.-X. Huang and Q.-B. Han, Nat. Prod. Rep., 2006, 23, 673-698.

16 R. W. Hoffmann, Term introduced by Hoffman in 2009 describing intermediates in a synthetic route that contain one or more $\mathrm{C}-\mathrm{C}$ bonds that will break to get the target product, Elements of Synthesis Planning, Springer, Berlin, 2009, pp. 106-117.

17 E. C. Cherney, J. C. Green and P. S. Baran, Angew. Chem., Int. Ed., 2013, 52, 9019-9022.

18 S. K. Nistanaki, L. A. Boralsky, R. D. Pan and H. M. Nelson, Angew. Chem., Int. Ed., 2019, 58, 1724-1726.

19 (a) Q. Zhou and B. B. Snider, Org. Lett., 2008, 9, 1401-1404 (synthesis of $( \pm$ )-vibralactone in 10 steps); $(b)$ Q. Zhou and B. B. Snider, J. Org. Chem., 2008, 73, 8049-8056 (synthesis of (-)-vibralactone in 11 steps); (c) A. J. Leeder, R. J. Heap, L. J. Brown, X. Franck and R. C. D. Brown, Org. Lett., 2016, 18, 5971-5973(synthesis of ( \pm )-vibralactone in 11 steps).
20 For instance: (a) K. I. Booker-Milburn, H. Jenkins, J. P. H. Charmant and P. Mohr, Org. Lett., 2003, 5, 3309-3312; (b) A. J. Blake, A. J. Highton, T. N. Majid and N. S. Simpkins, Org. Lett., 1999, 1, 1787-1789 and see references therein.

21 Y. Ito, S. Fujii and T. Saegusa, J. Org. Chem., 1976, 41, 2073-2074.

22 T. D. Michels, M. S. Dowling and C. D. Vanderwal, Angew. Chem., Int. Ed., 2012, 51, 7572-7576.

23 D. Niroula, L. P. Hallada, S. Rogelj and R. Tello-Aburto, Tetrahedron, 2017, 73, 359-364.

24 W.-W. Ren, Z.-X. Chen, Q. Xiao, Y. Li, T.-W. Sun, Z.-Y. Zhang, Q.-D. Ye, F.-K. Meng, L. You, M.-Z. Zhao, L.-M. Xu, Y.-F. Tang, J.-H. Chen and Z. Yang, Chem. - Asian J., 2012, 7, 2341-2350.

25 N. J. Truax, S. Ayinde, K. Van, J. O. Liu and D. Romo, Org. Lett., 2019, 21, 239-243.

26 See for instance: (a) K. Hattori, M. Nagano, T. Kato, I. Nakanishi, K. Imai, T. Kinoshita and K. Sakane, Bioorg. Med. Chem. Lett., 1995, 5, 2821-2824; (b) V. T. Tandon, K. A. Singh, A. K. Awasthi, J. M. Khanna, B. Lal and N. Anand, Bioorg. Med. Chem. Lett., 2004, 14, 2867-2870; (c) G. Britain and P. Press, Tetrahedron Lett., 1991, 32, 3083-3086.

27 Z. Zhao, A. J. To and G. K. Murphy, Chem. Commun., 2019, 55, 14821-14824.

28 (a) E. Carlson, R. Boutin and W. Tam, Tetrahedron, 2018, 74, 5510-5518; (b) C. Wicks, S. Koh, A. Pounder, E. Carlson and W. Tam, Tetrahedron Lett., 2019, 60, DOI: 10.1016/ j.tetlet.2019.151228.

29 X. Meng, M. Guo, J. Zhu, H. Zhu, X. Sun, L. Tian and Z. Cao, Eur. J. Org. Chem., 2019, 1952-1956.

30 See for instance the structure of Ingenol or the well-known Taxol.

31 P.-F. Li, C.-B. Yi and J. Qu, Org. Biomol. Chem., 2015, 13, 5012-5021.

32 For selected reviews on the synthesis of medium-size rings, see: (a) G. A. Molander, Acc. Chem. Res., 1998, 31, 603-609; (b) A. Deiters and R. Martin, Chem. Rev., 2004, 104, 2199-2238; (c) I. Shiina, Chem. Rev., 2007, 107, 239-273; (d) T. V. Nguyen, J. M. Hartmann and D. Enders, Synthesis, 2013, 845-873; (e) Y. Wang and Z.-W. Yu, Acc. Chem. Res., 2015, 48, 2288-2296; $(f)$ K. T. Mortensen, T. J. Osberger, T. A. King, H. F. Sore and D. R. Spring, Chem. Rev., 2019, 119, 10288-10317.

33 Y. Wang, W. Ju, H. Tian, S. Sun, W. Li, W. Tian and J. Gui, J. Am. Chem. Soc., 2019, 141, 5021-5033.

34 E. Stempel and T. Gaich, Acc. Chem. Res., 2016, 49, 2390-2402.

35 W.-L. Yang, W. Li, Z.-T. Yang and W.-P. Deng, Org. Lett., 2020, 22, 4026-4032.

36 D. Tymann, D. C. Tymann, U. Bednarzick, L. IovkovaBerends, J. Rehbein and M. Hiersemann, Angew. Chem., Int. Ed., 2018, 57, 15553-15557.

37 Y. Zhou, X. Tao, Q. Yao, Y. Zhao and Y. Li, Chem. - Eur. J., 2016, 22, 17936-17939. 
38 For a ring expansion strategy towards cyclohepta[b]chromene, see: J. L. C. Sousa and A. M. S. Silva, Synthesis, 2017, 316-322.

39 N. Ishida, S. Sawano and M. Murakami, Nat. Commun., 2014, 5, 3111-3119.

40 For a review on the subject, see: S. Kotha, M. E. Shirbhate and G. T. Waghule, Beilstein J. Org. Chem., 2015, 11, 1274-1331.

41 P. S. Baran, J. Am. Chem. Soc., 2018, 140, 4751-4755.

42 Z.-L. Song, C.-A. Fan and Y.-Q. Tu, Chem. Rev., 2011, 111, 7523-7556.

43 T. R. McDonald, L. R. Mills, M. S. West and S. A. L. Rousseaux, Chem. Rev., 2020, 121, 3-79.

44 (a) D. Li, W. Zang, M. J. Bird, C. J. T. Hyland and M. Shi, Chem. Rev., 2020, DOI: 10.1021/acs.chemrev.0c00624.; (b) G. Otárola, J. J. Vaquero, E. Merino and M. A. Fernández-Rodríguez, Catalysts, 2020, 10, DOI: 10.3390/catal10101178.

45 (a) M. Wang and P. Lu, Org. Chem. Front., 2018, 5, 254-259; (b) J. Li, K. Gao, M. Bian and H. Ding, Org. Chem. Front., 2020, 7, 136-154; (c) Y.-Y. Fan, X.-H. Gao and J.-M. Yue, Sci. China: Chem., 2016, 59, 1126-1141.

46 (a) E. Jiménez-Núñez, C. K. Claverie, C. Nieto-Oberhuber and A. M. Echavarren, Angew. Chem., Int. Ed., 2006, 45, 5452-5455; (b) S. G. Sethofer, S. T. Staben, O. Y. Hung and F. D. Toste, Org. Lett., 2008, 10, 4315-4318.

47 A. Pitaval, D. Leboeuf, J. Ceccon and A. M. Echavarren, Org. Lett., 2013, 15, 4580-4583.

48 A. Z. Wu, D. Leboeuf, P. Retailleau, V. Gandon, A. Marinetti and A. Voituriez, Chem. Commun., 2017, 53, 7026-7029.

49 J. P. Markham, S. T. Staben and F. D. Toste, J. Am. Chem. Soc., 2005, 127, 9708-9709.

50 W. Zang, L. Wang, Y. Wei, M. Shi and Y. Guo, Adv. Synth. Catal., 2019, 361, 2321-2328.

51 T. Matsuda and Y. Sakurai, J. Org. Chem., 2014, 79, 2739-2745.

52 B. Sahoo, J.-L. Li and F. Glorius, Angew. Chem., Int. Ed., 2015, 54, 11577-11580.

53 W.-D. Liu, G.-Q. Xu, X.-Q. Hu and F.-F. Xu, Org. Lett., 2017, 19, 6288-6291.

54 S. Y. Shim, Y. Choi and D. H. Ryu, J. Am. Chem. Soc., 2018, 140, 11184-11188.

55 (a) L. E. Overman and L. D. Pennington, J. Org. Chem., 2003, 68, 7143-7157; (b) X.-M. Zhang, Y.-Q. Tu, F.-M. Zhang, Z.-H. Chen and S.-H. Wang, Chem. Soc. Rev., 2017, 46, 2272-2305; (c) B.-M. Wang and Y.-Q. Tu, Acc. Chem. Res., 2011, 44, 1207-1222.

56 For selected examples on the semipinacol rearrangement, see: (a) D. H. Lukamto and M. J. Gaunt, J. Am. Chem. Soc., 2017, 139, 9160-9163; (b) C.-C. Xi, Z.-M. Chen, S.-Y. Zhang and Y.-Q. Tu, Org. Lett., 2018, 20, 4227-4230; (c) P. Natho, A. B. Rouse, J. L. Greenfield, L. A. T. Allen, A. J. P. White, Z. Yang and P. J. Parsons, Tetrahedron, 2020, 76, DOI: 10.1016/j.tet.2020.131636.

57 Y. Kim and Y. Kim, Asian J. Org. Chem., 2019, 8, 679-682.

58 (a) Y. Kim and D. Y. Kim, Tetrahedron Lett., 2019, 60, 1538-1542; (b) D. Y. Kim, Synth. Commun., 2019, 49, 2203-2209.
59 X.-F. Song, A.-H. Ye, Y.-Y. Xie, J.-W. Dong, C. Chen, Y. Zhang and Z.-M. Chen, Org. Lett., 2019, 21, 9550-9554.

60 Y.-Y. Xie, Z.-M. Chen, H.-Y. Luo, H. Shao, Y.-A. Tu, X. Bao, R. F. Cao, S.-Y. Zhang and J.-M. Tian, Angew. Chem., Int. Ed., 2019, 58, 12491-12496.

61 J.-C. Kang, Y.-Q. Tu, J.-W. Dong, C. Chen, J. Zhou, T. Ding, J.-T. Zai, Z.-M. Chen and S.-Y. Zhang, Org. Lett., 2019, 21, 2536-2540.

62 J. W. Park and D. Y. Kim, Bull. Korean Chem. Soc., 2019, 40, 1244-1247.

63 S.-H. Wang, B.-S. Li and Y.-Q. Tu, Chem. Commun., 2014, 50, 2393-2408.

64 (a) S. E. Denmark, D. J. P. Kornfilt and T. Vogler, J. Am. Chem. Soc., 2011, 133, 15308-15311; (b) E. Hartmann and S. E. Denmark, Helv. Chem. Acta, 2017, 100, eA700158.

65 J. Yang, X.-M. Zhang, F.-M. Zhang, S.-H. Wang, Y.-Q. Tu, Z. Li, X. C. Wang and H. Wang, Angew. Chem., Int. Ed., 2020, 59, 8471-8475.

66 H. Shao, K. Fang, Y.-P. Wang, X.-M. Zhang, T.-M. Ding, S.-Y. Zhang, Z.-M. Chen and Y.-Q. Tu, Org. Lett., 2020, 22, 3775-3779.

67 For a dearomative [2+2] cycloaddition/aza semipinacol ring expansion, see: J. Ma, F. Schäfers, C. Daniliuc, K. Bergander, C. A. Strassert and F. Glorius, Angew. Chem., Int. Ed., 2020, 59, 9639-9645.

68 L. Liu, L.-S. Lei, Z.-S. Zhan, S.-Z. Liu, Y.-X. Wang, Y.-Q. Tu, F.-M. Zhang, X.-M. Zhang, A.-J. Ma and S.-H. Wang, Chem. Commun., 2019, 55, 3789-3792.

69 F.-P. Zhu, X. Guo, F.-M. Zhang, X.-M. Zhang, H. Wang and Y.-Q. Tu, Org. Lett., 2020, 22, 2076-2080.

70 N. R. Candeias, R. Paterna and P. M. P. Gois, Chem. Rev., 2016, 116, 2937-2981.

71 W. Li, X. Liu, X. Hao, X. Hu, Y. Chu, W. Cao, S. Qin, C. Hu, L. Lin and W. Feng, J. Am. Chem. Soc., 2011, 133, 15268-15271.

72 (a) M. Dousset, K. Le Jeune, S. Cohen, J.-L. Parrain and G. Chouraqui, Synthesis, 2016, 2396-2401; (b) V. L. Rendina, H. Z. Kaplan and J. S. Kingsbury, Synthesis, 2012, 686-693; (c) H. Liu, C. Sun, N.-K. Lee, R. F. Henry and D. Lee, Chem. Eur. J., 2012, 18, 11889-11893.

73 (a) H. Cao, F. Chen, C. Su and L. Yu, Adv. Synth. Catal., 2020, 362, 438-461; (b) W. Fang and M. Shi, Chem. - Eur. J., 2018, 24, 9998-10005.

74 C.-G. Zhao, Z.-T. Feng, G.-Q. Xu, A. Gao, J.-W. Che, Z.-Y. Wang and P.-F. Xu, Angew. Chem., Int. Ed., 2020, 59, 3058-3062.

75 C.-L. Li and Z.-X. Yu, J. Org. Chem., 2019, 84, 9913-9928.

76 M. A. Beniddir, L. Evanno, D. Joseph, A. Skiredj and E. Poupon, Nat. Prod. Rep., 2016, 33, 820-842.

77 M. Hönig and E. M. Carreira, Angew. Chem., Int. Ed., 2020, 59, 1192-1196.

78 S. Eichenberger, L. Hönig, M. J. R. Richter, R. GershoniPoranne and E. Carreira, Chem. Sci., 2020, 11, 5294-5298.

79 (a) M. Kashiwabara, T. Kamo, H. Makabe, H. Shibata and M. Hirota, Biosci., Biotechnol., Biochem., 2006, 70, 1502-1505; (b) M. Hirota, Y. Shimizu, T. Kamo, H. Makabe and H. Shibata, Biosci., Biotechnol., Biochem., 2003, 67, 1597-1600. 
80 T. C. McMorris, A. Kashinatham, R. Lira, H. Rundgren, P. K. Gantzel, M. J. L. Kelmer and R. Dawe, Phytochemistry, 2002, 61, 395-398.

81 W. Ayer and M. H. Saeedi-Ghomi, Can. J. Chem., 1981, 59, 2536-2538.

82 D. Pan, Y. Wei and M. Shi, Org. Lett., 2017, 19, 3584-3587.

83 D. Pan, Y. Wei and M. Shi, Org. Chem. Front., 2019, 6, 1123-1232.

84 F. Yi, B. Huang, Q. Nie and M. Cai, Tetrahedron Lett., 2016, 57, 4405-4410.

85 Y. Chen, J. Wang, W. Li and A. Jia, Chin. J. Catal., 2013, 34, 1816-1819.

86 A. Simonneau, Y. Harrak, L. Jeanne-Julien, G. Lemière, V. Mouriès-Mansuy, J.-P. Goddard, M. Malacria and L. Fensterbank, ChemCatChem, 2013, 5, 1096-1099.

87 For selected examples of natural products, see: (a) E. M. Driggers, S. P. Hale, J. Lee and N. K. Terrett, Nat. Rev. Drug Discovery, 2008, 7, 608-624; (b) T. Tadross, S. Virgil and B. Stoltz, Org. Lett., 2010, 12, 1612-1614.

88 (a) A. Homs, C. Obradors, D. Lebœuf and A. M. Echavarren, Adv. Synth. Catal., 2014, 356, 221-228; (b) M. E. de Orbe, L. Amenós, M. S. Kirillova, Y. Wang, V. López-Carrillo, F. Maseras and A. M. Echavarren, J. Am. Chem. Soc., 2017, 139, 10302-10311.

89 M. E. de Orbe and A. M. Echavarren, Eur. J. Org. Chem., 2018, 2740-2752.

90 D.-F. Chen, P.-Y. Wu and L.-Z. Gong, Org. Lett., 2013, 15, 3958-3961.

91 (a) H. Xu, W. Zhang, D. Shu, J. B. Werness and W. Tang, Angew. Chem., Int. Ed., 2008, 47, 8933-8936; (b) J. M. Um, H. Xu, K. N. Houkand and W. Tang, J. Am. Chem. Soc., 2009, 131, 6664-6665For related studies, see: (c) J. Barluenga, L. Riesgo, L. A. Lápez, E. Rubio and M. Tomás, Angew. Chem., Int. Ed., 2009, 48, 7569-7572; (d) C.-W. Li, K. Pati, G.-Y. Lin, S. M. Abu Sohel, H.-H. Hung and R.-S. Liu, Angew. Chem., Int. Ed., 2010, 49, 9891-9894.

92 R. Liu, M. Zhang, T. P. Wyche, G. N. Winston-McPherson, T. S. Bugni and W. Tang, Angew. Chem., Int. Ed., 2012, 51, 7503-7506.

93 R. Liu, M. Zhang, G. G. N. Winston-McPherson and W. Tang, Chem. Commun., 2013, 49, 4376-4378.

94 D. P. Hari, J. C. Abell, V. Fasano and V. K. Aggarwal, J. Am. Chem. Soc., 2020, 142, 5515-5520.

95 (a) D. Garayalde and C. Nevado, Beilstein J. Org. Chem., 2011, 7, 767-780; (b) M. Shi, J. M. Lu, Y. Wei and L. X. Shao, Acc. Chem. Res., 2012, 45, 641-652.

96 E. Vogel, Angew. Chem., 1960, 72, 4-26.

97 (a) T. Hudlicky, R. Fan, J. W. Reed and K. G. Gadamasetti, Org. React., 1992, 4, 1-133; (b) S. Krüger and T. Gaïch, Beilstein J. Org. Chem., 2014, 10, 163-193.

98 I. Özkan and M. Zora, J. Org. Chem., 2003, 68, 9635-9642.

99 H. M. L. Davies, D. G. Stafford, B. D. Doan and J. H. Houser, J. Am. Chem. Soc., 1998, 120, 3326-3331.

100 H. M. L. Davies and J. R. Denton, Chem. Soc. Rev., 2009, 38, 3061-3071.

101 J. D. Osler, W. P. Unsworth and R. J. K. Taylor, Org. Biomol. Chem., 2013, 11, 7587-7594.
102 A. C. Wright, C. W. Lee and B. M. Stoltz, Org. Lett., 2019, 21, 9658-9662.

103 (a) R. A. Craig II, R. C. Smith, J. L. Roizen, A. C. Jones, S. C. Virgil and B. M. Stoltz, J. Org. Chem., 2018, 83, 3467-3485; (b) R. A. Craig II, R. C. Smith, J. L. Roizen, A. C. Jones, S. C. Virgil and B. M. Stoltz, J. Org. Chem., 2019, 84, 7722-7746; (c) R. A. Craig II, J. L. Roizen, R. C. Smith, A. C. Jones, S. C. Virgil and B. M. Stoltz, Chem. Sci., 2017, 8, 507-514.

104 J. L. Roizen, A. C. Jones, R. C. Smith, S. C. Virgil and B. M. Stoltz, J. Org. Chem., 2017, 82, 13051-13067.

105 A. Bartoli, G. Chouraqui and J.-L. Parrain, Org. Lett., 2012, 14, 122-125.

106 (a) W. Rao, Sally, S. N. Berry and P. W. H. Chan, Chem. Eur. J., 2014, 20, 13174-13180; (b) S. Y. Kim, Y. Park and Y. K. Chung, Angew. Chem., Int. Ed., 2010, 49, 415-418.

107 E. T. Newcomb, P. C. Knutson, B. A. Pedersen and E. M. Ferreira, J. Am. Chem. Soc., 2015, 138, 108-111.

108 Y.-J. Wang, X.-X. Li and Z. Chen, J. Org. Chem., 2020, 85, 7694-7703.

109 For related recent examples not detailed in the main text, see: (a) H. Wang, C.-Y. Zhou and C.-M. Che, Adv. Synth. Catal., 2017, 359, 2253-2258; (b) Y.-J. Chiang and J.-L. Zhu, Tetrahedron Lett., 2017, 58, 3081-3084; (c) R. Kats-Kagan and S. B. Herzon, Org. Lett., 2015, 17, 2030-2033.

110 (a) S. E. Reisman, R. R. Nani and S. Levin, Synlett, 2011, 2437-2442; (b) T. Ye and M. A. McKervey, Chem. Rev., 1994, 94, 1091-1160; (c) C. A. Merlic and A. L. Zechman, Synthesis, 2003, 1137-1156; (d) A. Ford, H. Miel, A. Ring, C. N. Slattery, A. R. Maguire and M. A. McKervey, Chem. Rev., 2015, 115, 9981-10080.

111 For a recent example, see the total synthesis of Salvilucalin C: C. Fu, Y. Zhang, C. Zhu, B. Wang and H. Ding, Org. Lett., 2014, 16, 3376-3379.

112 T. Hoshi, E. Ota, Y. Inokuma and J. Yamaguchi, Org. Lett., 2019, 21, 10081-10084.

113 For examples on Cope rearrangements of divinylcyclobutanes, see: (a) M. L. Snapper, J. A. Tallarico and M. L. Randall, J. Am. Chem. Soc., 1997, 119, 1478-1479 and references therein; (b) P. A. Wender and C. R. D. Correia, J. Am. Chem. Soc., 1987, 109, 2523-2525; (c) P. C.-K. Lo and M. L. Snapper, Org. Lett., 2001, 3, 2819-2821.

114 For recent examples, see: (a) A. E. Hurtley, Z. Lu and T. P. Yoon, Angew. Chem., Int. Ed., 2014, 53, 8991-8994; (b) S. Yamada, Y. Azuma and K. Aya, Tetrahedron Lett., 2014, 55, 2801-2804.

115 (a) A. Vázquez-Sánchez and J. G. Ávila-Zárraga, Tetrahedron Lett., 2017, 58, 981-984; (b) H. Tan, C. Zheng, Z. Liu and S. Z. Wang, Org. Lett., 2011, 13, 2192-2195.

116 T. Truong and O. Daugulis, Chem. Sci., 2013, 4, 531-535.

117 Y. Yamamoto, K.-I. Nishimura, S. Mori and M. Shibuya, Angew. Chem., Int. Ed., 2017, 56, 5494-5497.

118 T. Miura, T. Nakamuro, C.-J. Liang and M. Murakami, J. Am. Chem. Soc., 2014, 136, 15905-15908.

119 (a) D. Mal and S. R. De, Org. Lett., 2009, 11, 4398-4401; (b) M. A. Ernst-Russell, C. L. Chai, J. H. Wardlaw and 
J. A. Elix, J. Nat. Prod., 2000, 63, 129-131; (c) A. K. Khalafallah, J. Chin. Chem. Soc., 2001, 48, 801-806; (d) J. B. Scaglione, B. D. Manion, A. Benz, A. Taylor, G. T. DeKoster, N. P. Rath, A. S. Evers, C. F. Zorumski, S. Mennerick and D. F. Covey, J. Med. Chem., 2006, 49, 4595-4605; (e) E. Benedetti, A. B. Veliz, M. Charpenay, L. S. Kocsis and K. M. Brummond, Org. Lett., 2013, 15, 2578-2581; $(f)$ Y. Hu, H. Yao, Y. Sun, J. Wan, X. Lin and T. Zhu, Chem. - Eur. J., 2010, 16, 7635-7641.

120 H.-Z. Wei, L. Z. Yu and M. Shi, Org. Biomol. Chem., 2020, 18, 135-139.

121 P. A. Clarke, S. Santos and W. H. C. Martin, Green. Chem., 2007, 9, 438-440.

122 (a) B. Schäfer, Naturstoffe der Chemischen Industrie, Elsevier, München, 2007; (b) T. Maki and K. Takeda, Ullmann's Encyclopedia of Industrial Chemistry, Wiley-VCH, Weinheim, 2011.

123 (a) J. Feierfeil, A. Grossmann and T. Magauer, Angew. Chem., Int. Ed., 2015, 54, 11835-11838; (b) J. Feierfeil and T. Magauer, Chem. - Eur. J., 2018, 24, 1455-1458.

124 For selected examples, see: (a) F. A. Carey and R. A. Sundberg, Advanced Organic Chemistry, Springer, Heidelberg, 2007, pp. 1003-1056; (b) F. Terrier, Modern Nucleophilic Aromatic Substitution, Wiley-VCH, Weinheim, 2013; (c) M. G. Weaver, W.-J. Bai, S. K. Jackson and T. R. R. Pettus, Org. Lett., 2014, 16, 1294-1297; (d) S. Valente, Z. Xu, E. Bana, C. Zwergel, A. Mai, C. Jacob, P. Meiser, D. Bagrel, A. M. S. Silva and G. Kirsch, Eur. J. Org. Chem., 2013, 2869-2877; (e) S. Giroux and E. J. Corey, Org. Lett., 2008, 10, 5617-5619.

125 M. Schmid, A. S. Grossmann, P. Meyer, T. Müller and T. Magauer, Tetrahedron, 2019, 75, 3195-3215.

126 T. A. Unzner, A. S. Grossmann and T. Magauer, Angew. Chem., Int. Ed., 2016, 55, 9763-9767.

127 (a) H. W. Moore and O. H. W. Decker, Chem. Rev., 1986, 86, 821-830; (b) S. T. Perri, S. D. Foland, O. H. W. Decker and H. W. Moore, J. Org. Chem., 1986, 51, 3067-3068; (c) O. H. W. Decker and H. W. Moore, J. Org. Chem.,
1987, 52, 1174-1175; (d) M. W. Reed and H. W. Moore, J. Org. Chem., 1987, 52, 3491-3492; (e) L. D. Foland, J. O. Karlsson, S. T. Perri, R. Schwabe, S. L. Xu, S. Patil and H. W. Moore, J. Am. Chem. Soc., 1989, 111, 975-989; $(f)$ A. Enhsen, K. Karabelas, J. M. Heerding and H. W. Moore, J. Org. Chem., 1990, 55, 1177-1185; $(g)$ Y. Xiong and H. W. Moore, J. Org. Chem., 1996, 61, 9168-9177.

128 (a) L. S. Liebeskind, S. Iyer and C. F. Jewell, J. Org. Chem., 1986, 51, 3065-3067; (b) L. S. Liebeskind, Tetrahedron, 1989, 45, 3053-3060.

129 S. Gao and X. Hu, Org. Chem. Front., 2017, 4, 1493-1498.

130 Z. Ren, Y. Hao and X. Hu, Org. Lett., 2016, 18, 4958-4961.

131 For another example of 4-electron/6 6 -electrocyclisation sequence reported during the time-period covered by this review, see: V. Ramadoss, A. J. Alonso-Castro, N. CamposXolalpa and C. Solorio-Alvarado, J. Org. Chem., 2018, 83, 10627-10635.

132 N. Arichi, K.-I. Yamada, Y. Yamaoka and K. Takasu, J. Am. Chem. Soc., 2015, 137, 9579-9582.

133 X.-N. Wang, E. H. Krenske, R. C. Johnston, K. N. Houk and R. P. Hsung, J. Am. Chem. Soc., 2015, 137, 5596-5601.

134 For references on the construction of quaternary centres, see: (a) K. W. Quasdorf and L. E. Overman, Nature, 2014, 516, 181-191; (b) J. J. Murphy, D. Bastida, S. Paria, M. Fagnoni and P. Melchiorre, Nature, 2016, 532, 218-222; (c) S. A. Green, T. R. Huffman, R. O. McCourt, V. van der Puyl and R. A. Shenvi, J. Am. Chem. Soc., 2019, 141, 7709-7714; (d) J. T. Mohr, D. C. Behenna, A. M. Harned and B. M. Stoltz, Angew. Chem., Int. Ed., 2005, 44, 6924-6927; (e) P.-W. Xu, J.-S. Yu, C. Chen, Z.-Y. Cao, F. Zhou and J. Zhou, ACS Catal., 2019, 9, 1820-1882; $(f)$ H. Wu, Q. Wang and J. Zhu, J. Am. Chem. Soc., 2019, 141, 11372-11377; $(g)$ J.-Y. Liu, J. Zhao, J.-L. Zhang and P.-F. Xu, Org. Lett., 2017, 19, 1846-1849; (h) T. Zhu, Y. Liu, M. Smetankova, S. Zhuo, C. Mou, H. Chai, Z. Jin and Y. R. Chi, Angew. Chem., Int. Ed., 2019, 58, 15778-15782. 